\title{
PSEUDOHOLOMORPHIC CURVES AND THE SHADOWING LEMMA
}

\author{
KAI CIELIEBAK AND ERIC SÉRÉ
}

1. Introduction. Let $M$ be a compact smooth manifold of dimension $n$, and let $T^{*} M \rightarrow^{\tau} M$ be its cotangent bundle. $T^{*} M$ carries a canonical 1-form $\theta$, which in canonical coordinates $\left(q_{i}, p_{i}\right)$ is given by

$$
\theta=\sum p_{i} d q_{i}
$$

Then $\omega:=d \theta$ is a symplectic form on $T^{*} M$.

To a smooth Hamiltonian $H \in C^{\infty}\left(S^{1} \times T^{*} M, \mathbb{R}\right), 1$-periodic in time, we associate the Hamiltonian system

$$
\dot{x}=X_{H}(t, x),
$$

where the Hamiltonian vector field $X_{H}$ is defined by

$$
d_{x} H(t, x)=\omega\left(X_{H}(t, x), \cdot\right) .
$$

We make the following assumptions on $H$.

(H1) (Saddle point). There exists a point $x_{0}=\left(q_{0}, 0\right) \in T^{*} M$ such that $H\left(t, x_{0}\right)=0$, $d_{x} H\left(t, x_{0}\right)=0$ for all $t$, and

$$
\begin{aligned}
& \frac{\partial^{2} H}{\partial p^{2}}\left(t, x_{0}\right)_{p p}>0 \quad \text { for all } t, \quad H\left(t, q_{0}, p\right) \geq 0 \text { for all } t, p, \\
& \frac{\partial^{2} H}{\partial q^{2}}\left(t, x_{0}\right)_{q q}<0 \quad \text { for all } t, \quad H(t, q, 0)<0 \quad \text { for all } q \neq q_{0} .
\end{aligned}
$$

(H2) (Growth conditions). We have that

(i) $\left|d_{x} H(t, x)\right| \leq \operatorname{ad}\left(x, x_{0}\right)$;

(ii) $H(t, q, p) \geq b_{1}|p|^{2}-b_{2}$;

(iii) there exists a vector field $\eta$ on $T^{*} M$ satisfying

$$
\begin{aligned}
& d\left(i_{\eta} \omega\right)=\omega, \\
& |\eta(x)| \leq c_{1} d\left(x, x_{0}\right), \\
& d_{x} H(t, x) \cdot \eta(x)-H(t, x) \geq c_{2}\left(d\left(x, x_{0}\right)\right)^{2} .
\end{aligned}
$$

Here, $a, b_{i}, c_{i}$ are positive constants; we have chosen a Riemannian metric on $M$ and 
denoted by $|\cdot|$ and $d(\cdot, \cdot)$ the induced metric and distance on $T^{*} M$. Note, however, that $(\mathrm{H} 2)$ does not depend on the choice of metric.

Assumption (H1) implies, in particular, that $x_{0}$ is a hyperbolic equilibrium of the Hamiltonian system (HS). We explain this in the comments at the end of the introduction. Examples of Hamiltonians satisfying $(\mathrm{H} 1),(\mathrm{H} 2)$, and references to earlier works are also given in these comments. Now, let

$$
\mathscr{C}=\left\{x \in C^{\infty}(\mathbb{R}, T M) \mid \dot{x}=X_{H}(t, x), \lim _{t \rightarrow \pm \infty} x(t)=x_{0}\right\}
$$

be the set of all solutions of (HS) that are doubly asymptotic to $x_{0}$. The elements of $\mathscr{C} \backslash\left\{x_{0}\right\}$ are called orbits homoclinic to $x_{0}$. Since $H$ is 1 -periodic in time, the integers act on $\mathscr{C}$ via

$$
\begin{aligned}
*: \mathbf{Z} \times \mathscr{C} & \rightarrow \mathscr{C} \\
(n, x) & \rightarrow n * x(t)=x(t-n) .
\end{aligned}
$$

In [6], the following result is proved.

THEOREM 1.1. Assume that (H1), (H2) are satisfied. Then there are infinitely many orbits homoclinic to $x_{0}$, which are in different classes of $\mathscr{C} / \mathbf{Z}$.

The homoclinic orbits are found as critical points of an action functional. The proof uses the pseudoholomorphic curves introduced by Gromov and Floer (see [13], [11], [14], [15], [5]).

We point out that the hypotheses on $H$ in [6] are slightly stronger than (H1), (H2). But it is not very difficult to weaken these assumptions, as we see at the end of Section 5 .

Now near a homoclinic orbit, one expects, under certain assumptions, to find chaotic behavior. This goes back to Poincaré, who observed in 1899 that in the neighborhood of a homoclinic orbit, there may exist an infinite number of further homoclinic orbits giving rise to a very complicated orbit structure: "On sera frappé de la complexité de cette figure, que je ne cherche même pas à tracer" (see [19, p. 387]). Later, this was made precise by Birkhoff, Smale, Silnikov, and others in terms of symbolic dynamics. Recall the definition of a Bernoulli shift. Let

$$
\Sigma=\{0,1\}^{\mathbf{Z}}
$$

be the set of all doubly infinite sequences endowed with the metric

$$
d(a, b)=\sum_{n \in \mathbf{Z}} \frac{\left|b_{n}-a_{n}\right|}{2^{|n|}} .
$$

The Bernoulli shift is given by the homeomorphism

$$
\begin{aligned}
\sigma: & \Sigma \rightarrow \Sigma \\
& \left(a_{n}\right)_{n \in \mathbf{N}} \rightarrow\left(a_{n+1}\right)_{n \in \mathbf{N}} .
\end{aligned}
$$


We say that a homeomorphism $\phi$ on an invariant subset $A$ is semiconjugate to a Bernoulli shift if there exists a continuous surjection $\tau: A \rightarrow \Sigma$ such that the following diagram commutes:

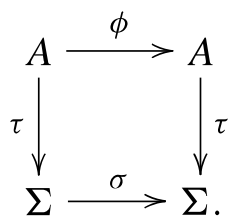

This is conjugate to a Bernoulli shift if $\tau$ is a homeomorphism.

It is a classical result that if $x_{0}$ is a hyperbolic fixed point (or periodic point) of a diffeomorphism $\phi$, and the stable and unstable manifolds of $x_{0}$ have a transverse intersection outside $x_{0}$, then there exists a set $A$ on which the iterate $\phi^{N}$ is conjugate to a Bernoulli shift, for $N \in \mathbf{N}$ sufficiently large (see, for instance, [18]).

However, it is quite unnatural to presuppose tranversality of orbits that are to be found by variational methods. Instead we use a weaker global hypothesis, as follows.

(C) Any connected component of $\mathscr{C}$ for the $H^{1,2}\left(\mathbb{R}, T^{*} M\right)$-topology is compact for this topology.

Here we use an embedding $T^{*} M \subset \mathbb{R}^{2 a}$, identifying $x_{0}$ with zero, to define the Hilbert manifold $H^{1,2}\left(\mathbb{R}, T^{*} M\right)$.

THEOREM 1.2. If $H$ satisfies (H1), (H2), and $(\mathrm{C})$, then for each sufficiently large $T \in \mathbf{N}$, there exists a compact subset $A_{T} \subset T^{*} M$, invariant under the time- $T$ map $\phi_{T}$ of(HS), such that $\phi_{T}$ is semiconjugate to a Bernoulli shift on $A_{T}$.

The rate at which a system is chaotic can be measured by the topological entropy as defined by Bowen (see [27, pp. 182-183]):

$$
h_{\text {top }}(\phi)=\sup _{R>0} \lim _{\epsilon \rightarrow 0}\left(\limsup _{n \rightarrow \infty} \frac{\ln s(n, \epsilon, R)}{n}\right) \text {, }
$$

where

$$
\begin{aligned}
s(n, \epsilon, R)=\max \{ & \operatorname{card}(E) \mid E \subset B(0, R), \\
& \left.(\forall x \neq y \in E)(\exists k \in[0, n]):\left|\phi^{k}(x)-\phi^{k}(y)\right| \geq \epsilon\right\} .
\end{aligned}
$$

Theorem 1.2 and the fact that the entropy of a Bernoulli shift is $\ln (2)$ immediately imply the following corollary.

Corollary 1.3. If(H1), (H2), and $(\mathrm{C})$ are true, then the time-1 map $\phi_{1}$ has a positive topological entropy.

The presence of a Bernoulli shift near a transverse homoclinic orbit is usually derived from the so-called shadowing lemma, which states that, near an approximate solution, one finds a real one.

Theorem 1.2 also follows from a kind of shadowing lemma. However, since the usual proof of the shadowing lemma relies heavily on the transversality assumption 
(which allows one to use the contraction mapping principle), we cannot expect the shadowing lemma to hold in its classical form. Rather, we obtain a "topological shadowing lemma," as we now explain.

Using an isometric embedding $J: M \rightarrow \mathbb{R}^{a}$ such that $J\left(q_{0}\right)=0$, we talk of norms $|x|$ and differences $x-y$ for $x, y$ in $T^{*} M \subset \mathbb{R}^{2 a}$ ( $x_{0}$ is identified with zero).

Let $x_{i}: \mathbb{R} \rightarrow T^{*} M$ be given for $1 \leq i \leq p$, and let $R_{i}>0$ be such that $\left|x_{i}(t)\right|$ is smaller than the injectivity radius $r_{0}$ of $M$ outside the interval $\left[-R_{i}, R_{i}\right]$. If $n_{i} \in \mathbf{Z}$ are such that $n_{i+1}-n_{i} \geq R_{i}+R_{i+1}+2$, then, using cutoff functions, one can define the "multibump" function $\sum n_{i} * x_{i}$ (see [6]). This function coincides with $n_{i} * x_{i}$ on $\left[n_{i}-R_{i}, n_{i}+R_{i}\right]$, and $\left|\sum n_{i} * x_{i}(t)\right| \leq \max _{1 \leq i \leq p}\left|x_{i}\left(t-n_{i}\right)\right|<r_{0}$ outside these intervals. Our main theorem follows.

TheOREM 1.4 (Topological shadowing). Let $M, H$ be as above, and let (H1), (H2), and $(C)$ be satisfied. Then we can find a compact set $\mathscr{C}_{0} \subset \mathscr{C} \backslash\left\{x_{0}\right\}$, and, for every $\epsilon>0$, an integer $N(\epsilon)>0$ with the following property.

If $\left(n_{i}\right)_{i \in I}$ is a family of integers, the index set I being either $\mathbf{Z}, \mathbf{N},-\mathbf{N}$, or $\{1, \ldots, l\}$, and $n_{i+1}-n_{i} \geq 2 N(\epsilon)$, then there exist $y_{i} \in \mathscr{C}_{0}$ and a solution $y$ of $\dot{y}=X_{H}(t, y)$ such that

$$
\begin{aligned}
& \left|y_{i}(t)-x_{0}\right| \leq \epsilon \quad \text { for }|t| \geq N(\epsilon)-1, \\
& \left|y(t)-\sum_{i \in I} n_{i} * y_{i}(t)\right| \leq \epsilon \quad \text { for all } t \in \mathbb{R} .
\end{aligned}
$$

Moreover, the following hold:

if $I= \pm \mathbf{N}$, then $y(t) \rightarrow x_{0}$ as $t \rightarrow \mp \infty$;

if $I=\{1, \ldots, l\}$, then $y$ is a homoclinic orbit "with l bumps;"

if $I=\mathbf{Z}$ and $\left(n_{i}\right)$ is periodic, that is, $n_{i+p}=n_{i}+q$ for some $p, q \in \mathbf{N}$ and all

$i \in \mathbf{Z}$, then $y$ may be chosen periodic, ofperiod $q$.

In contrast to the classical versions of the shadowing lemma, we cannot prescribe precisely which $y_{i}$ are to occur. This is due to the fact that in the proof, we "glue together" sets of orbits supporting certain cohomology classes rather than individual orbits. But the structure we find is rich enough to give Theorem 1.2.

ProofofTheorem 1.2 as a consequence ofTheorem 1.4. Since $\mathscr{C}_{0}$ is compact and does not contain $x_{0}$, there is $\epsilon>0$ such that $\left|y(0)-x_{0}\right| \geq 4 \epsilon$ for all $y \in \mathscr{C}_{0}$. Let $T=2 N(\epsilon)$. For each $n \in \mathbf{Z}$, the map that associates $\phi_{n T}(x) \in T M$ to each $x \in T M$ is continuous. Hence

$$
A_{T}=\left\{x \in T M:\left|\phi_{n T}(x)-x_{0}\right| \in[0,2 \epsilon] \cup[3 \epsilon, R], \forall n \in \mathbf{Z}\right\}
$$

is compact, where $R>\sup \left\{\left|y(0)-x_{0}\right|: y \in \mathscr{C}_{0}\right\}+\epsilon$.

Clearly $A_{T}$ is $\phi_{T}$-invariant. Moreover, the map $\tau: A_{T} \rightarrow \Sigma=\{0,1\}^{\mathbf{Z}}$, defined by

$$
[\tau(x)]_{n}=1 \quad \text { if }\left|\phi_{n T}(x)-x_{0}\right| \geq 3 \epsilon,
$$




$$
[\tau(x)]_{n}=0 \quad \text { if }\left|\phi_{n T}(x)-x_{0}\right| \leq 2 \epsilon,
$$

is continuous; one readily checks that $\tau \circ \phi_{T}=\sigma \circ \tau$, where $\sigma$ is the Bernoulli shift on $\Sigma$; that is, $\sigma\left(\left(a_{n}\right)_{n \in \mathbf{Z}}\right)=\left(a_{n+1}\right)_{n \in \mathbf{Z}}$.

For the surjectivity of $\tau$, let $\left(a_{n}\right) \in \Sigma$ be given. Write the set $\left\{n \in \mathbf{Z}: a_{n}=1\right\}$ in increasing order as $\left(k_{i}\right)_{i \in I}$, where $I$ is chosen as in Theorem 1.4 according to whether $\left\{n \in \mathbf{Z}: a_{n}=1\right\}$ is unbounded, bounded from below, and/or bounded from above. (The choice of $\left(k_{i}\right)$ is not unique in the case of a doubly infinite sequence.)

By the choice of $T$, the sequence $\left(k_{i} T\right)_{i \in I}$ satisfies the hypothesis of Theorem 1.4, with $\epsilon$ as above. Hence there exists a solution $y$ and $y_{i} \in \mathscr{C}_{0}$ such that

$$
\left|y(t)-\sum_{i \in I}\left(k_{i} T\right) * y_{i}\right| \leq \epsilon, \quad \forall t \in \mathbb{R} .
$$

Let $x=y(0)$. If $n \in \mathbf{Z}$ with $a_{n}=1$, we have

$$
\begin{aligned}
\left|\phi_{n T}(x)-x_{0}\right| & =\left|y(n T)-x_{0}\right| \\
& \geq\left|\sum_{i \in I}\left(k_{i} T\right) * y_{i}(n T)-x_{0}\right|-\epsilon \\
& =\left|y_{j}(0)-x_{0}\right|-\epsilon \geq 3 \epsilon,
\end{aligned}
$$

where $k_{j}=n$. Hence $[\tau(x)]_{n}=1=a_{n}$. Similarly, if $a_{n}=0$, then

$$
\left|\phi_{n T}(x)-x_{0}\right| \leq \max _{i}\left|y_{i}\left(\left(n-k_{i}\right) T\right)-x_{0}\right|+\epsilon \leq 2 \epsilon,
$$

and hence $[\tau(x)]_{n}=0$. This proves the equality $\tau(x)=\left(a_{n}\right)_{n \in \mathbf{Z}}$.

Comments. (1) The hypothesis (H1) implies that $x_{0}$ is a hyperbolic fixed point of the time-1 map $\phi_{1}$ of (HS); that is,

$$
D \varphi_{1}\left(x_{0}\right): T_{x_{0}} T^{*} M \rightarrow T_{x_{0}} T^{*} M
$$

has no eigenvalue of modulus 1 . Indeed, the linearized system at $x_{0}$ is of the form

$$
\dot{q}=A(t) p, \quad \dot{p}=B(t) q
$$

in $\mathbb{R}^{n} \times \mathbb{R}^{n}$, with both matrices $A, B$ positive definite and 1-periodic in time. If $D \varphi_{1}\left(x_{0}\right)$ has an eigenvalue of modulus 1 , then this system has a nonzero quasiperiodic solution $(p(t), q(t))$. Then we write

$$
p \cdot q(T)-p \cdot q(0)=\int_{0}^{T} p \cdot \dot{q}+q \cdot \dot{p}=\int_{0}^{T} p \cdot A(t) p+q \cdot B(t) q .
$$

But as $T$ goes to infinity, $p \cdot q(T)$ remains bounded, whereas the integral on the right-hand side goes to infinity, a contradiction. 
(2) As a consequence of (H2), the energy levels $\left\{x \in T^{*} M ; H(t, x)=h\right\}$ are of contact type, for all $t \in S^{1}, h>0$.

(3) The hypotheses (H1), (H2) are symplectically invariant in the following sense. Let $\psi: T^{*} M \rightarrow T^{*} M$ be a symplectomorphism mapping the zero section onto itself, mapping the fibre $T_{q_{0}}^{*} M$ onto the fibre $T_{\psi\left(q_{0}\right)}^{*} M$ (here we identify $q \in M$ with $(q, 0) \in$ $\left.T^{*} M\right)$, and satisfying

$$
\frac{1}{a}|v| \leq|D \psi(x) \cdot v| \leq a|v| \quad \text { for all }(x, v) \in T\left(T^{*} M\right) .
$$

Then a Hamiltonian $H(t, x)$ satisfies (H1), (H2) with saddle point $x_{0}$ if and only if $H\left(t, \psi^{-1}(x)\right)$ satisfies (H1), (H2) with saddle point $\psi\left(x_{0}\right)$.

(4) The hypotheses (H1), (H2) are satisfied, in particular, by classical Hamiltonians on the cotangent bundle of a compact Riemannian manifold $M$,

$$
H(t, q, p)=\frac{|p-A(t, q)|^{2}}{2}+V(t, q)
$$

with the following properties:

(j) $V$ has a unique nondegenerate absolute maximum $q_{0}$; that is,

$$
\begin{aligned}
& V\left(t, q_{0}\right)=0, \quad d_{q} V\left(t, q_{0}\right)=0, \quad \frac{\partial^{2} V}{\partial q^{2}}\left(t, q_{0}\right)<0 \\
& \text { (ji) } \begin{cases}A\left(t, q_{0}\right)=0, & \\
\left|d_{q} A\left(t, q_{0}\right) \cdot \xi\right|^{2}+\left\langle\frac{\partial^{2} V}{\partial q^{2}}\left(t, q_{0}\right) \cdot \xi, \xi\right\rangle<0, & \forall \xi \in T_{q_{0}} M, \\
\frac{1}{2}|A(t, q)|^{2}+V(t, q)<0 & \text { for any } q \neq q_{0} .\end{cases}
\end{aligned}
$$

In this case, the vector field $\eta$ of $(\mathrm{H} 2)$ is defined by $\omega(\eta, \cdot)=\theta$. In canonical coordinates, $\eta(q, p)=(0, p)$.

Homoclinics for this kind of Hamiltonian were first studied variationally by Bolotin [3] and later by several authors (see, e.g., [20], [1]). In these works, the convexity of $H$ in $p$ is essential: The homoclinics are found as critical points of a Lagrangian functional. To our knowledge, the first generalization to nonconvex Hamiltonians is due to Felmer [10] (existence of $2 n$ homoclinics when $M$ is the $n$-torus).

(5) There has been a lot of work in recent years on variational gluing of homoclinic orbits (see, e.g., [22], [8], [24], [9], [2], [12], [4], [21], [17]). In these works, the Hamiltonian presents some convexity, so that either a Lagrangian functional or a dual action functional (see [7]) can be used. A first homoclinic orbit is found by mountain pass or Ljusternik-Shnirelman theory; then a gluing method introduced by Séré [22] is used to get an analogue of Theorem 1.4. In the present paper, the main novelty is that no convexity assumption is made on the Hamiltonian. 
(6) The first variational results on Bernoulli shift associated to homoclinics were obtained under assumptions slightly stronger than (C). In [23], the hypothesis is as follows.

$(\mathscr{H}) \mathscr{C}$ is at most countable.

Recently, a condition similar to (C) was introduced by Rabinowitz [21] in the context of singular Lagrangian systems in $\mathbb{R}^{2}$. Note that for some classes of lowdimensional Hamiltonian systems, multibump solutions can be constructed, assuming only that $W^{u} \neq W^{s}$ (see [2], [4], [17]).

2. Sketch of the proof of Theorem 1.4. From now on, we fix a Riemannian metric on $M$. It induces an isomorphism $T M \simeq T^{*} M$, which allows us to transfer all structures such as $\omega, H$, and $\theta$ to $T M$. Without changing notation, we henceforth work with $T M$ instead of $T^{*} M$. We call $M_{0}$ the zero section of $T M$.

Theorem 1.4 is proved by a refinement of the method used in [6]. It is based on the variational principle for the action functional

$$
\begin{gathered}
I: H^{1,2}(\mathbb{R}, T M) \rightarrow \mathbb{R} \\
I(x):=-\int_{\mathbb{R}} x^{*}\left(i_{\eta} \omega\right)-\int_{\mathbb{R}} H(t, x(t)) d t .
\end{gathered}
$$

Here we have used an isometric embedding $T M \hookrightarrow \mathbb{R}^{2 a}$, mapping $x_{0}$ to zero, to define Sobolev classes $H^{m, p}$ for mappings into $T M$. So $x \in H^{1,2}(\mathbb{R}, T M)$ implies in particular that $x(t) \rightarrow x_{0}$ as $t \rightarrow \pm \infty$.

The critical points of $I$ are exactly the elements of $\mathscr{C}$. Let $I^{\prime}(x)$ be the $L^{2}$-gradient of $I$ defined by

$$
d I(x) \xi=\left\langle I^{\prime}(x), \xi\right\rangle_{L^{2}} \text { for all } \xi \in H^{1,2}\left(\mathbb{R}, x^{*} T M\right) .
$$

With the help of the almost complex structure $J$ on $T M$ defined by

$$
\omega(J \cdot, \cdot)=\langle\cdot, \cdot\rangle,
$$

we can write $I^{\prime}(x)$ explicitly as

$$
I^{\prime}(x)=-J(x) \dot{x}-H^{\prime}(t, x) .
$$

Hence the equation of gradient lines $u: \mathbb{R}^{2} \rightarrow T M, u_{s}=I^{\prime}(u(s))$, becomes the inhomogeneous nonlinear Cauchy-Riemann equation

$$
\bar{\partial} u+H^{\prime}(t, u) \equiv u_{s}(s, t)+J(u(s, t)) u_{t}(s, t)+H^{\prime}(t, u(s, t))=0 .
$$

Its solutions are called "pseudoholomorphic curves." They were introduced by Gromov [13] and Floer [11].

Now fix a $T>1$ and define

$$
\Omega_{T}^{\infty}:=\left\{\bar{q} \in C^{\infty}(\mathbb{R}, M) \mid \bar{q}(t) \equiv q_{0} \text { for }|t| \geq T\right\},
$$


equipped with the $C^{\infty}$-topology. For $n=\left(n_{1}, \ldots, n_{p}\right) \in \mathbf{Z}^{p}$ such that $n_{i+1}-n_{i} \geq 2 T$, let

$$
n * \Omega_{T}^{\infty}:=\left\{\sum_{i=1}^{p} n_{i} * q_{i} \mid q_{i} \in \Omega_{T}^{\infty}\right\} .
$$

For $R>1$ and $\bar{q} \in n * \Omega_{T}^{\infty}$, we study the set

$$
\begin{gathered}
X_{\bar{q}, R}:=\left\{u \in H^{2,2}([-R, R] \times \mathbb{R}, T M) \mid u(-R, t) \in M_{0},\right. \\
\left.u(R, t) \in T_{\bar{q}(t)} M, \text { and } \bar{\partial} u+H^{\prime}(t, u)=0\right\} .
\end{gathered}
$$

The elements of $X_{\bar{q}, R}$ are the solutions of an elliptic boundary value problem, and by standard regularity arguments, they are smooth functions of $(s, t)$. From the variational viewpoint, $X_{\bar{q}, R}$ should be seen as the set of finite gradient lines connecting the space of curves in the zero section $M_{0}$ to the space of curves over $\bar{q}$. Note that the zero section and the fibres are Lagrangian submanifolds of $(T M, \omega)$ transverse to each other.

In [6], it was shown that $X_{\bar{q}, R}$ is nonempty for every $\bar{q}$ and $R$. The crucial point now is to find elements $u \in X_{\bar{q}, R}$ that have the same "multibump shape" as $\bar{q}=\sum_{i=1}^{p} n_{i} * q_{i}$ in the following sense. Given $\epsilon>0$, if $(\forall i) n_{i+1}-n_{i} \geq 2 N(\epsilon)$, then there are $p$ pseudoholomorphic curves $u_{i} \in X_{q_{i}, R}$, such that

$$
\left|u(s, t)-u_{i}(s, t)\right| \leq \epsilon \quad \text { for all }(s, t) \in[-R, R] \times\left[\frac{n_{i-1}+n_{i}}{2}, \frac{n_{i}+n_{i+1}}{2}\right] .
$$

To have this estimate independent of $R$, we use assumption (C). Then we can let $R \rightarrow \infty$ to obtain a space $X_{\infty}$ of infinite gradient lines of "multibump shape." Their asymptotics for $s \rightarrow \infty$ are multibump homoclinic orbits of $x_{0}$. Finally, from the fact that $X_{\infty}$ carries a nontrivial product cohomology, we conclude that there must be at least one homoclinic orbit with all bumps nontrivial.

Section 3 deals with some consequences of (C) on the compactness of Palais-Smale sequences. In Section 4, we prove the basic estimate on finite gradient lines; and in Section 5, Theorem 1.4 is proved. Both sections make extensive use of results in [6]. Since these results have been proved under the assumption that $\mathscr{b} / \mathbf{Z}$ is finite, we show how the proofs must be modified under the weaker assumption $(C)$.

Acknowledgements. This work is the sequel of [6], which was based on a suggestion by $\mathrm{H}$. Hofer. The authors wish to thank $\mathrm{H}$. Hofer for his encouragement and for fruitful conversations. A part of this paper was written while the second author was visiting the Courant Institute at New York University. He thanks the members of the Courant Institute, particularly J. Shatah, for their kind hospitality.

3. Some consequences of (C). We first introduce some notation. Given $h>0$, we denote $\mathscr{C}^{h}=\{x \in \mathscr{C}, I(x) \leq h\}$. For $\alpha>0, x, y \in \mathscr{C}^{h}$, we write $x \sim_{\alpha} y$ when 
there is a finite sequence $x=x_{0}, x_{1}, \ldots, x_{n}=y$ in $\mathscr{C}^{h}$ such that $\left\|x_{i+1}-x_{i}\right\|_{L^{2}(\mathbb{R})}<\alpha$ for all $i$.

Here, the distance $\|x-y\|_{L^{2}(\mathbb{R})}$ is defined using an isometric embedding $T M \hookrightarrow$ $\mathbb{R}^{2 a}$. Note that the choice of an $L^{2}$-distance in this definition is not crucial, because the $L^{2}$-distance is equivalent to the $H^{1,2}$-distance on $\mathscr{G}^{h}$ (but not to the $L_{\text {loc }}^{2}$-distance).

We see that $\sim_{\alpha}$ is obviously an equivalence relation on $\mathscr{G}^{h}$. Its equivalence classes are called the $\alpha$-connected components of $\mathscr{C}^{h}$. If $A$ and $B$ are two distinct classes, then $d(A, B) \geq \alpha$ by construction. Here, we have used the standard notation

$$
d(A, B)=\inf \left\{\|x-y\|_{L^{2}} ;(x, y) \in A \times B\right\} .
$$

The following lemma is a consequence of Sard's theorem.

Lemma 3.1. Assume that (H1), (H2) are satisfied. Let $F$ be the set ofsums ofthe form $I\left(u_{1}\right)+\cdots+I\left(u_{p}\right)$ with $p$ arbitrary, and let $u_{1}, \ldots, u_{p} \in \mathscr{C}$.

(i) For any $h>0$ and $\eta>0$, there exists $\zeta(h, \eta)$ such that, if $J$ is an interval of size $\eta$ included in $[0, h]$, then there is an interval $J^{\prime}$ of size $\zeta$ included in $J \cap(\mathbb{R} \backslash F)$.

(ii) $I$ is constant on each connected component of $\mathscr{C}$.

(iii) For any $h>0$ and $\eta>0$, there exists $\alpha(h, \eta)$ such that, if $\Gamma_{\alpha} \subset \mathscr{G}^{h}$ is $\alpha$ connected, then

$$
\sup I\left(\Gamma_{\alpha}\right)-\inf I\left(\Gamma_{\alpha}\right)<\eta .
$$

Proof. (i) Sard's theorem cannot be applied to $I: H^{1} \rightarrow \mathbb{R}$ since $H^{1}$ is infinitedimensional. So we perform local finite-dimensional reductions. Let $X$ be a critical point of $I$. By definition, $X(0)$ is in the intersection of the unstable and stable manifolds $W^{u}, W^{s}$ of $x_{0}$ for the time-1 map of the Hamiltonian system. Let $L^{u}, L^{s}$ be the tangent spaces to $W^{u}, W^{s}$ at $X(0)$. $W^{u}, W^{s} \subset T M$ are Lagrangian manifolds, so $L^{u}, L^{s} \subset T_{X(0)}(T M)$ are Lagrangian planes. We choose a Lagrangian plane $L_{X} \subset T_{X(0)}(T M)$ transverse to $L^{u}$ and $L^{s}$. Let $U \subset T_{X(0)}\left(T^{*} M\right)$ be an open ball with center at the null vector $O$ and with radius $r$. Taking $r$ small enough, one can construct a symplectomorphism $\phi$, from $U$ to an open neighborhood $V$ of $X(0)$ in $T M$, such that $\phi(O)=X(0), D \phi(O)$ is the identity, and $\phi\left(L^{u} \cap U\right) \subset W^{u}$. Let $w^{s}$ be an embedded Lagrangian submanifold of $U$ containing $O$ and such that $\phi\left(w^{s}\right) \subset W^{s}$. The tangent plane to $w^{s}$ at $O$ is $L^{s}$.

By transversality of $L_{X}$ with $L^{u}, L^{s}$, for $U^{\prime} \subset U$ a small enough neighborhood of $O$, there is a smooth mapping $z: U^{\prime} \cap L^{u} \rightarrow w^{s}$ such that $z(y)-y \in L_{X}$ for any $y \in$ $U^{\prime} \cap L^{u}$. Then we can define $x_{-}(t), t \leq 0$, as the unique solution of the Hamiltonian system such that $x_{-}(t) \rightarrow x_{0}$ as $t \rightarrow-\infty$ and $x_{-}(0)=\phi(y)$. Similarly, $x_{+}(t), t \geq 0$, is the unique solution such that $x_{+}(t) \rightarrow x_{0}$ as $t \rightarrow+\infty$ and $x_{+}(0)=\phi \circ z(y)$.

Since $L_{X}$ is Lagrangian, there is a system of canonical variables $(Q, P)$ in $T_{X(0)}$ (TM) such that $L_{X}$ has equation $Q=0$ and $L^{u}$ has equation $P=0$. Let $\alpha=\phi^{*}\left(i_{\eta} \omega\right)$. The form $P d Q-\alpha$ is closed on the ball $U$. Therefore it is exact, and there is a smooth function $f$ from $T M$ to $\mathbb{R}$ such that $P d Q=\alpha+d(f \circ \phi)$ on $U$, and $f\left(x_{0}\right)=0$. We denote $\beta=i_{\eta} \omega+d f$. 
Let

$$
\begin{aligned}
A_{-}(y) & =\int_{-\infty}^{0}-x_{-}^{*}(\beta)-H\left(t, x_{-}(t)\right) d t \\
& =-f \circ \phi(y)+\int_{-\infty}^{0}-x_{-}^{*}\left(i_{\eta} \omega\right)-H\left(t, x_{-}(t)\right) d t
\end{aligned}
$$

be the action of $x_{-}$; similarly,

$$
\begin{aligned}
A_{+}(y) & =\int_{0}^{+\infty}-x_{+}^{*}(\beta)-H\left(t, x_{+}(t)\right) d t \\
& =f \circ \phi(z)+\int_{0}^{+\infty}-x_{-}^{*}\left(i_{\eta} \omega\right)-H\left(t, x_{-}(t)\right) d t .
\end{aligned}
$$

We define a smooth mapping $j: U^{\prime} \cap L^{u} \rightarrow \mathbb{R}$ by $j(y)=A_{-}(y)+A_{+}(y)$.

We claim that

$$
j^{\prime}(y) d y=-P(Q) d Q
$$

with $y=(Q, 0), d y=(d Q, 0)$, and $z(y)=(Q, P(Q))$. So $y$ is a critical point of $j$ if and only if $z(y)=y$.

To see this, consider the extended phase space $\mathbb{R} \times S^{1} \times T M$ with coordinates $(h, t, x)$ and the symplectic form $\omega+d h \wedge d t=d(\beta+h d t)$. Let $W_{t}^{u, s}$ be the unstable, respectively, stable manifolds of $x_{0}$ for the time-1 map of the Hamiltonian flow starting at time $t$. The manifolds

$$
\hat{W}^{u, s}:=\left\{(h, t, x) \mid t \in S^{1}, x \in W_{t}^{u, s}, h=H(t, x)\right\} \subset \mathbb{R} \times S^{1} \times T M
$$

are Lagrangian submanifolds for $\omega+d h \wedge d t$.

Asymptotic solutions $x_{\mp}(t)$ as above define curves $t \mapsto\left(H(t, x), t, x_{\mp}(t)\right)$ in $\hat{W}^{u, s}$. It follows that for $y, y^{\prime} \in U^{\prime} \cap L^{u}$,

$$
\begin{aligned}
& A_{-}\left(y^{\prime}\right)-a_{-}(y)=-\int_{y}^{y^{\prime}} \phi^{*}(\beta+H d t)=0, \\
& A_{+}\left(y^{\prime}\right)-A_{+}(y)=-\int_{z(y)}^{z\left(y^{\prime}\right)} \phi^{*}(\beta+H d t)=-\int_{z(y)}^{z\left(y^{\prime}\right)} \beta,
\end{aligned}
$$

where the integrals are taken along any paths from $y$ to $y^{\prime}$ in $U^{\prime} \cap L^{u}$, respectively, from $z(y)$ to $z\left(y^{\prime}\right)$ in $w^{s}$. Here we have used that, along these paths, $d t=0$ and $\left.\phi^{*} \beta\right|_{L^{u}}=0$. Hence for $y=(Q, 0) \in U^{\prime} \cap L^{u}, v=(V, 0) \in L^{u}$, and $\epsilon>0$ small,

$$
\begin{aligned}
j(y+\epsilon v)-j(y) & =-\int_{z(y)}^{z(y+\epsilon v)} \phi^{*} \beta \\
& =-\int_{(Q, P(Q))}^{(Q+\epsilon V, P(Q+\epsilon V))} P d Q=-\int_{0}^{\epsilon} P(Q+s V) \cdot V d s .
\end{aligned}
$$


This shows that $j^{\prime}(y) \cdot v=-P(Q) \cdot V$, and the claim is proved.

Now, there is a neighborhood $\mathscr{V}_{X}$ of $X$ in $H^{1,2}(\mathbb{R}, T M)$ such that the critical levels of $I$ in $\mathscr{V}_{X}$ are also critical levels of $j$. Indeed, if $x$ is close to $X$ in $H^{1,2}$, then $x(0)$ is close to $X(0)$ and there is a unique $y \in U^{\prime} \cap L^{u}$ such that $x(0)=\phi(y)$. If $x$ is a homoclinic orbit, we also have $x(0)=\phi \circ z(y)$ so that $y$ is a critical point of $j$. Then $x$ coincides with $x_{-}$on $\mathbb{R}_{-}$and with $x_{+}$on $\mathbb{R}_{+}$; hence $j(y)=I(x)$.

The family $\left(\mathscr{V}_{X}\right)_{X \in \mathscr{C}}$ is an open covering of the set $\mathscr{C} \subset H^{1,2}(\mathbb{R}, T M)$ of critical points of $I$. Since $H^{1,2}(\mathbb{R}, T M)$ is paracompact, we can find a locally finite refinement $\left(\mathcal{U}_{\lambda}\right)_{\lambda \in \Lambda}$ of $\left(\mathscr{V}_{X}\right)_{X \in \mathscr{C}}$. For $\lambda \in \Lambda$, let $X_{\lambda}$ be such that $\mathcal{u}_{\lambda} \subset \mathscr{V}_{X_{\lambda}}$. We call $j_{\lambda}$ the associated finite-dimensional functional, we call $D_{\lambda}$ its $n$-dimensional domain, and we call $K_{\lambda}$ the set of its critical levels. By Sard's theorem, $K_{\lambda}$ has measure zero. Since $H^{1,2}$ is separable, we can achieve that $\Lambda$ is at most countable. So $I(\mathscr{C})=\bigcup_{\lambda \in \Lambda} K_{\lambda}$ has measure zero.

Given $\lambda_{1}, \lambda_{2} \in \Lambda$, the function $J_{\lambda_{1}, \lambda_{2}}: D_{\lambda_{1}} \times D_{\lambda_{2}} \rightarrow \mathbb{R}$ defined by $J_{\lambda_{1}, \lambda_{2}}\left(y_{1}, y_{2}\right)=$ $j_{\lambda_{1}}\left(x_{1}\right)+j_{\lambda_{2}}\left(x_{2}\right)$ is smooth and defined on a domain of dimension $2 n$. So the set $K_{\lambda_{1}}+K_{\lambda_{2}}$ of critical values of $J_{\lambda_{1}, \lambda_{2}}$ has measure zero. As a consequence, $I(\mathscr{C})+$ $I(\mathscr{C})=\bigcup_{\left(\lambda_{1}, \lambda_{2}\right) \in \Lambda \times \Lambda} K_{\lambda_{1}}+K_{\lambda_{2}}$ has measure zero. More generally, the set $F$ of sums of the form $I\left(u_{1}\right)+\cdots+I\left(u_{p}\right), u_{i} \in \mathscr{C}, p$ arbitrary, has measure zero. But $F$ is closed by concentration-compactness (see [23, Lemma 5] and [6, Lemma 2.5]). So $\mathbb{R} \backslash F$ is a dense open subset of $\mathbb{R}$. By compactness of $[0, h]$, we finally obtain the existence of the uniform gap $\zeta(h, \eta)$.

(ii) The image of a connected component of $\mathscr{b}$ under the continuous map $I$ is connected; it is thus an interval included in $F$. But $\mathbb{R} \backslash F$ is dense, so this interval is a point.

(iii) Since $I$ is uniformly continuous on $\mathscr{C}^{h}$, if $\Gamma^{\alpha}$ is $\alpha$-connected, then $I\left(\Gamma_{\alpha}\right)$ is $\beta$-connected, where $\beta$ is small for $\alpha$ small. Take $\beta=\zeta(h, \eta) / 2$. We cannot have $x, y \in \Gamma_{\alpha}$ with $I(x) \geq I(y)+\eta$. Otherwise, taking $J=[I(y), I(y)+\eta]$, we could find $x_{0}, \ldots, x_{N}$ with $I\left(x_{k+1}\right)-I\left(x_{k}\right) \leq \zeta / 2$; this would contradict (i).

If an $\alpha$-connected component $\Gamma_{\alpha} \subset \mathscr{b}^{h}$ is compact, then $\Gamma_{\alpha} \cap n * \Gamma_{\alpha}=\emptyset$ for any nonzero integer $n$; hence $\operatorname{dist}\left(\Gamma_{\alpha}, n * \Gamma_{\alpha}\right) \geq \alpha$.

Let us introduce the following assumptions.

$\left(C_{\alpha}\right)$ For any $h>0$, there exists $\alpha(h)>0$ such that when $\alpha \leq \alpha(h)$, all the $\alpha$-connected components of $\mathscr{C}^{h}$ are compact for the $H^{1,2}(\mathbb{R}, T M)$-topology.

$\left(C_{\Lambda}\right)$ There does not exist a set $\Lambda \subset T M$ with the following properties:

(i) $\Lambda$ is a subset of $W_{\mathrm{loc}}^{s} \cap W^{u}$ or $W_{\mathrm{loc}}^{u} \cap W^{s}$;

(ii) $x_{0} \in \Lambda$ and $\Lambda \neq\left\{x_{0}\right\}$;

(iii) $\Lambda$ is compact and connected, and the solutions of the Hamiltonian system having a point of $\Lambda$ as initial condition are in $\mathscr{b}$, their action being less than or equal to some fixed number $h_{0}$.

Remark 1. Assumption $\left(C_{\Lambda}\right)$ is similar to assumption (1.10) in the work of Rabinowitz [21]. 
Remark 2. In the case where $M=S^{1}, W^{u} \backslash\left\{x_{0}\right\}$ is a disjoint union $W_{+}^{u} \cup W_{-}^{u}$ of two smooth curves. In the same way, $W^{s} \backslash\left\{x_{0}\right\}=W_{+}^{s} \cup W_{-}^{s}$. If $\left(C_{\Lambda}\right)$ is not satisfied, then one of the curves $W_{ \pm}^{u}$ coincides with one of the curves $W_{ \pm}^{s}$. This shows that $\left(C_{\Lambda}\right)$ is weaker and easier to check than transversality. Let us recall that Bessi [2] (perturbative case) and Montechiarri-Nolasco-Terracini [17] (global case) found multibump solutions of the 1-dimensional pendulum, assuming only that $W^{u} \neq W^{s}$. See also Buffoni-Séré [4], for a similar assumption in the case of autonomous, realanalytic Hamiltonian systems in dimension 4. Theorem 1.2 can be considered as a generalization of [2] and [17].

Lemma 3.2. Assume that (H1), (H2) are satisfied. Then $(C),\left(C_{\alpha}\right)$, and $\left(C_{\Lambda}\right)$ are equivalent.

Proof. $\quad\left(\mathbf{C}_{\alpha}\right) \Rightarrow(\mathbf{C})$. We denote by $\Gamma_{\alpha}(x)$ the $\alpha$-connected component of $x \in \mathscr{C}^{h}$. If $\alpha<\beta$, then $\Gamma_{\alpha}(x) \subset \Gamma_{\beta}(x)$. If $\left(C_{\alpha}\right)$ is true, then $\tilde{\Gamma}(x)=\bigcap_{\alpha>0} \Gamma_{\alpha}(x)$ is compact, connected, and hence is contained in the connected component $\Gamma(x)$ of $x$ in $\mathscr{b}$. On the other hand, by Lemma 3.1, $I \equiv h$ on $\Gamma(x)$; this implies that $\Gamma(x) \subset \Gamma_{\alpha}(x)$ for all $\alpha$; hence $\Gamma(x)=\tilde{\Gamma}(x)$ is compact.

$\left(\mathbf{C}_{\Lambda}\right) \Rightarrow\left(\mathbf{C}_{\alpha}\right)$. We recall that $T M$ is identified as a submanifold of some space $\mathbb{R}^{2 a}$ with induced metric, and the equilibrium point $x_{0}$ is identified with zero. Suppose that $\left(C_{\alpha}\right)$ is false. Then there exist $h_{0}>0$, and for each positive integer $n$, there exists a sequence $\left(x_{p}^{n}\right)_{p \geq 0}$ in $\mathscr{C}^{h_{0}}$ such that

(a) $\left\|x_{p+1}^{n}-x_{p}^{n}\right\|_{H^{1,2}(\mathbb{R})} \leq 1 / n$ for all $p \geq 0$;

(b) the sequence $\left(x_{p}^{n}\right)_{p \geq 0}$ is not precompact; that is, it has a subsequence with no converging subsequence in $H^{1,2}(\mathbb{R}, T M)$.

Since $x_{0}$ is a hyperbolic equilibrium, there is a number $\bar{r}>0$ with the following property: If a forward orbit $\left\{\varphi^{n}(x) ; n \geq 0\right\}$ is entirely contained in the ball $B_{\bar{r}}\left(x_{0}\right)$, then $\left\{\varphi^{n}(x) ; n \geq 0\right\} \subset W_{\text {loc }}^{s}\left(x_{0}\right)$ and an analogous statement holds for backward orbits and $W_{\text {loc }}^{u}\left(x_{0}\right)$. In particular, any homoclinic orbit must leave $B_{\bar{r}}\left(x_{0}\right)$.

Now consider for fixed $n>1 / \bar{r}$ the sequence $\left(x_{p}^{n}\right)_{p \geq 0}$ as described above. Choose $\tilde{\tau}_{n} \in \mathbf{N}$ large enough such that $x_{0}^{n}(k) \leq 1 / n$ for all integers $\mathrm{k}$ such that $|k| \geq \tilde{\tau}_{n}$.

By concentration-compactness (see [16] or [6, Lemma 2.5]), since $\left(x_{p}^{n}\right)$ is not precompact, there must be a "bump" running away at infinity in time. More precisely, there are two sequences of integers $\tau^{n}, P^{n}$ with $\left|\tau^{n}\right| \geq \tilde{\tau}_{n}, P^{n}>0$, and such that

(1) $\left|x_{p}^{n}(k)\right| \leq \bar{r}$ for $p<P^{n}$ and $|k| \geq\left|\tau^{n}\right|$;

(2) $\left|x_{P^{n}}^{n}(k)\right| \leq \bar{r}$ for $|k|>\left|\tau^{n}\right|$;

(3) $\left|x_{P^{n}}^{n}\left(\tau^{n}\right)\right| \geq \bar{r}$.

By the choice of $\tau^{n}$, we have moreover that

(4) $\left|x_{0}^{n}\left(\tau^{n}\right)\right| \leq 1 / n$.

After extracting a subsequence, we may assume that all $\tau^{n}$ have the same sign, say, all $\tau^{n}$ are positive.

Let $\Lambda$ be the set of cluster points of $\left\{x_{p}^{n}\left(\tau^{n}\right) ; n>1 / \bar{r}, 0 \leq p \leq P^{n}\right\}$. 
The positivity of $\tau^{n}$ together with (1) and the choice of $\bar{r}$ implies that $\Lambda_{0} \subset$ $W_{\text {loc }}^{s}\left(x_{0}\right)$. From the compactness of $\mathscr{C}^{h_{0}}$ in the $H_{\text {loc }}^{1,2}$-topology, we get that all points of $\Lambda$ are initial data of orbits in $\mathscr{C}^{h_{0}}$. In particular, $\Lambda \subset W^{u}\left(x_{0}\right)$. If the $\tau_{n}$ are negative, we obtain the other possibility, $\Lambda \subset W_{\text {loc }}^{u} \cap W^{s}$.

From (4), it follows that $x_{0} \in \Lambda$, and from (3), $\Lambda$ contains a point on the boundary of $B_{\bar{r}}\left(x_{0}\right)$; hence $\Lambda \neq\left\{x_{0}\right\}$. Moreover, $\Lambda$ is $\alpha$-connected for any $\alpha>0$ and thus is connected.

$(\mathbf{C}) \Rightarrow\left(\mathbf{C}_{\Lambda}\right)$. Suppose that $(C)$ is true but $\left(C_{\Lambda}\right)$ is false. Let $\Lambda$ satisfy (i)-(iii) of $\left(C_{\Lambda}\right)$. Let $0: \Lambda \rightarrow \mathscr{C}^{h_{0}}$ be the function that associates with each $\lambda$ the unique orbit $\mathrm{O}_{\lambda}(\cdot)$ such that $\mathrm{O}_{\lambda}(0)=\lambda$. We also define $\mathscr{A}(\lambda)=I\left(\mathcal{O}_{\lambda}\right)$.

Applying concentration-compactness again, we see that $\mathscr{A}$ is continuous at a point $\lambda_{0}$ if and only if $\mathcal{O}$ is continuous at this point, with the $H^{1,2}(\mathbb{R}, T M)$-topology for the target. Moreover, if $\mathscr{A}$ is not continuous at $\lambda_{0}$, then

$$
\limsup _{\lambda \rightarrow \lambda_{0}} \mathscr{A}(\lambda) \geq \mathscr{A}\left(\lambda_{0}\right)+\rho
$$

where $\rho>0$ is independent of $\lambda_{0}$ (see [6, Lemma 2.3] for a choice of $\rho$ ). Let $\mathscr{L} \subset \Lambda$ be the set of all points where $\mathscr{A}, \mathcal{O}$ are continuous. By construction, we have

$$
\sup \mathscr{A}(\Lambda) \leq h_{0}
$$

so if we fix $\lambda_{*} \in \Lambda$ such that

$$
\mathscr{A}\left(\lambda_{*}\right) \geq \sup \mathscr{A}(\Lambda)-\frac{\rho}{2}
$$

then $\lambda_{*} \in \mathscr{L}$. Let $\mathscr{L}_{*}$ be the connected component of $\lambda_{*}$ in $\mathscr{L}$.

Since $\mathcal{O}$ is continuous on $\mathscr{L}$, the set $\mathcal{O}\left(\mathscr{L}_{*}\right)$ is connected for the $H_{\mathrm{glob}}^{1,2}$-topology. Let $\Gamma$ be the connected component of $\mathcal{O}\left(\mathscr{L}_{*}\right)$ in $\mathscr{C}$. By assumption $(\mathrm{C}), \Gamma$ is compact in $H^{1,2}(\mathbb{R}, T M)$. We infer that there is $\varepsilon_{*}>0$ such that if

$$
x(\cdot) \in \mathscr{C} \quad \text { and } \quad \inf _{y \in \Gamma}|x(0)-y(0)| \leq \varepsilon_{*},
$$

then $I(x) \geq I(\Gamma)-\rho / 4 \geq \sup \mathscr{A}(\Lambda)-3 \rho / 4$.

Otherwise, we would find sequences $\left(x_{n}\right) \subset \mathscr{C}$ and $\left(y_{n}\right) \subset \Gamma$ with $\left|x_{n}(0)-y_{n}(0)\right| \rightarrow$ 0 and $I\left(x_{n}\right) \leq I(\Gamma)-(\rho / 4)$. Then $\left(x_{n}\right)$ converges in $H_{\text {loc }}^{1,2}$ to $x \in(\mathscr{b})$ with $I(x) \leq$ $I(\Gamma)-(\rho / 4)$, and $\left(y_{n}\right)$ converges in $H^{1,2}$ to $y \in \Gamma$. But $x(0)=y(0)$ implies that $x=y$; hence there is a contradiction.

So the neighborhood $\mathscr{L}_{*}\left(\varepsilon_{*}\right)=\left\{\lambda \in \Lambda\right.$; $\left.\operatorname{dist}\left(\lambda, \mathscr{L}_{*}\right) \leq \varepsilon_{*}\right\}$ is included in $\mathscr{L}$; that is, 0 is continuous on $\mathscr{L}_{*}\left(\varepsilon_{*}\right)$. For $\alpha>0$, we denote by $\gamma_{\alpha}$ the $\alpha$-connected component of $\mathscr{L}_{*}$ in $\mathscr{L}_{*}\left(\varepsilon_{*}\right)$. Each $\gamma_{\alpha}$ is closed in $\Lambda$, and hence is compact, so $\bigcap_{\alpha>0} \gamma_{\alpha}$ is connected. But $\mathscr{L}_{*} \subset \bigcap_{\alpha>0} \gamma_{\alpha} \subset \mathscr{L}$, so $\mathscr{L}_{*}=\bigcap_{\alpha>0} \gamma_{\alpha}$, and the Hausdorff distance $d_{H}\left(\gamma_{\alpha}, \mathscr{L}_{*}\right)$ tends to zero as $\alpha$ goes to zero.

Now, let $\alpha$ be such that $\gamma_{\alpha} \subset \mathscr{L}_{*}\left(\varepsilon_{*}-\alpha\right)$. We denote $A=\gamma_{\alpha}, B=\Lambda \backslash \gamma_{\alpha}$. 
We have $\left|\lambda-\lambda^{\prime}\right| \geq \alpha$ whenever $\lambda \in A, \lambda^{\prime} \in B$. Indeed, either $\lambda^{\prime} \in \mathscr{L}_{*}\left(\varepsilon_{*}\right)$ and this comes from the definition of $A$, or $\lambda^{\prime} \notin \mathscr{L}_{*}\left(\varepsilon_{*}\right)$ and this comes from $\gamma_{\alpha} \subset \mathscr{L}_{*}\left(\varepsilon_{*}-\alpha\right)$. This implies that $A$ and $B$ are both open and closed.

$A$ contains $\lambda_{*}$, so it is nonempty. But $\Lambda$ is connected, so $B$ must be empty, and therefore $\mathscr{L}=\Lambda$. Thus $O$ is continuous on $\Lambda$, and $O(\Lambda)$ is connected in $H^{1,2}(\mathbb{R}, T M)$. So Lemma 3.1 implies that $\mathscr{A}$ is constant on $\Lambda$. But $\Lambda$ contains zero as well as a nonzero point $\bar{\lambda}$, and $\mathscr{A}(0)=0<\mathscr{A}(\bar{\lambda})$. This is a contradiction, and the lemma is proved.

Now assume that (C) is true, and take $h>0, \alpha \leq \alpha(h)$, with the notation of $\left(C_{\alpha}\right)$. Since $H^{1,2}$ is separable and two different $\alpha$-connected components have distance at least $\alpha$, there is an at most countable family $\left(Q_{\alpha}^{j}\right)_{j \in J_{\alpha}}$ of $\alpha$-connected components of $\mathscr{G}^{h}$ such that the following hold.

(1) For any $j$, there is $x_{j} \in Q_{\alpha}^{j}$ such that $\left|x_{j}(0)-x_{0}\right|>\bar{r}$, with some fixed $\bar{r}>0$.

(2) Any $\alpha$-connected component of $\mathscr{C}^{h}$ is of the form $n * Q_{\alpha}^{j}$, for some $n \in \mathbf{Z}$ and $j \in J_{\alpha}$.

By compactness, for any $j \in J_{\alpha}$, there is $\Delta(j)>0$ such that $\left|x(t)-x_{0}\right|<$ $r_{0} e^{-\chi(|t|-\Delta(j))}$ for any $x \in Q_{\alpha}^{j}$ and $|t| \geq \Delta(j)$. Here, $r_{0}$ is the injectivity radius of $M$ and $\chi$ is the smallest positive real part of the Floquet multipliers at $x_{0}$.

Now, given $\varepsilon>0$, a positive integer $p$, and a $p$-tuple $\bar{j}=\left(j_{1}, \ldots, j_{p}\right) \in\left(J_{\alpha}\right)^{p}$, we call $Z^{p}(\bar{j}, \varepsilon)$ the set of $p$-tuples $\bar{n}=\left(n_{1}, \ldots, n_{p}\right) \in \mathbf{Z}^{p}$ such that $n_{k+1}-n_{k} \geq$ $\Delta\left(j_{k}\right)+\Delta\left(j_{k+1}\right)+1 / \varepsilon$ for all $k$. For $0<\varepsilon<r_{0}$ and $\bar{j}=\left(j_{1}, \ldots, j_{p}\right) \in\left(J_{\alpha}\right)^{p}$, $\bar{n}=\left(n_{1}, \ldots, n_{p}\right) \in Z^{p}(\bar{j}, \varepsilon)$, let

$$
\Gamma_{\alpha}^{p}(\bar{j}, \bar{n})=n_{1} * Q_{\alpha}^{j_{1}}+\cdots+n_{p} * Q_{\alpha}^{j_{p}} .
$$

These sets can be defined using cutoff functions (see [6]), because each function $x(t)$ in $Q_{\alpha}^{j_{k}}$ satisfies $\left|x(t)-x_{0}\right|<r_{0}$ for any $|t| \geq \Delta\left(j_{k}\right)$.

For $1 \leq i \leq p$, let

$$
I_{i}=\left[\frac{n_{i-1}+n_{i}}{2}, \frac{n_{i+1}+n_{i}}{2}\right] .
$$

Define

$$
U_{\alpha}^{p}(\bar{j}, \bar{n}, \varepsilon)=\left\{x \in H^{1,2} ; \text { dist }_{H^{1,2}\left(I_{i}\right)}\left(\left.x\right|_{I_{i}},\left.n_{i} * Q_{\alpha}^{j_{i}}\right|_{I_{i}}\right) \leq \varepsilon \text { for all } 1 \leq i \leq p\right\} .
$$

Note that any $x$ in $\mathscr{C} \backslash\left\{x_{0}\right\}$ satisfies $I(x) \geq \rho$ (see [6, Lemma 2.3]). Let $h$ b e fixed as above and $p>h / \rho$ be an integer. Choosing $\Delta_{j}$ large enough, we can achieve

$$
\|x\|_{H^{1,2}\left(|t| \geq \Delta_{j}\right)} \leq \delta
$$

for all $j \in J_{\alpha}$ and $x \in Q_{\alpha}^{j}$.

Then for every $x \in U_{\alpha}^{p}(\bar{j}, \bar{n}, \varepsilon)$, we have

$$
I\left(\left.x\right|_{I_{i}}\right) \geq I\left(\left.x_{i}\right|_{I_{i}}\right)-c \varepsilon \geq \rho-\delta-c \varepsilon
$$


with $x_{i} \in Q_{\alpha}^{j_{i}}$, and therefore

$$
I(x) \geq p(\rho-\delta-c \varepsilon)>h,
$$

if $\delta$ and $\varepsilon$ are sufficiently small. Hence in this case,

$$
U^{p}(\bar{j}, \bar{n}, \varepsilon) \cap I^{h}=\emptyset .
$$

Now, let

$$
\begin{gathered}
G_{\alpha}(\varepsilon)=\bigcup_{1 \leq p \leq \frac{h}{\rho}, \bar{j} \in\left(J_{\alpha}\right)^{p}, \bar{n} \in Z^{p}(\bar{j}, \varepsilon)} \Gamma_{\alpha}^{p}(\bar{j}, \bar{n}), \\
V_{\alpha}(\varepsilon)=\bigcup_{1 \leq p \leq \frac{h}{\rho}, \bar{j} \in\left(J_{\alpha}\right)^{p}, \bar{n} \in Z^{p}(\bar{j}, \varepsilon)} U_{\alpha}^{p}(\bar{j}, \bar{n}, \varepsilon) .
\end{gathered}
$$

We are ready to state the analogue of [6, Corollary 2.6].

Lemma 3.3. Assume that (H1), (H2), and (C) are satisfied. We fix $h>0, \alpha \leq \alpha(h)$, and we consider the sets $V_{\alpha}(\varepsilon)$ defined above. For any $\varepsilon>0$, there is $\mu(\varepsilon)>0$ such that if

$$
I(x) \leq h \quad \text { and } \quad\left\|I^{\prime}(x)\right\|_{L^{2}} \leq \mu
$$

then $x \in V_{\alpha}(\varepsilon)$.

Proof. One argues by contradiction. If Lemma 3.3 is false, then for some $h_{0}, \varepsilon_{0}>$ 0 there is a sequence $\left(x_{n}\right)$ in $H^{1,2}$ such that $I\left(x_{n}\right) \leq h_{0}$ and $I^{\prime}\left(x_{n}\right) \rightarrow 0, x_{n} \notin V_{\alpha}\left(\varepsilon_{0}\right)$. We apply the concentration-compactness lemma to $x_{n}$ (see [16] and [6, Proposition 2.5]) and get a contradiction.

The next proposition is the analogue of [6, Lemma 2.7]. It is an immediate consequence of $(\mathrm{C})$.

Lemma 3.4. Assume that (H1), (H2), and (C) are satisfied. We take $h>0, \alpha \leq$ $\alpha(h)$, and we consider the sets $\Gamma_{\alpha}^{p}(\bar{j}, \bar{n})$ defined above.

There is $\varepsilon(\alpha)>0$ such that, if $\varepsilon \leq \varepsilon(\alpha), p_{1}, p_{2} \geq 1, \overline{j_{k}} \in\left(J_{\alpha}\right)^{p_{k}}, \overline{n_{k}} \in Z^{p_{k}}\left(\overline{j_{k}}, \varepsilon\right)$ for $k=1,2$, and $\left(p_{1}, \overline{j_{1}}, \overline{n_{1}}\right) \neq\left(p_{2}, \overline{j_{2}}, \overline{n_{2}}\right)$, then

$$
\operatorname{dist}_{L^{2}}\left(\Gamma_{\alpha}^{p_{1}}\left(\overline{j_{1}}, \overline{n_{1}}\right), \Gamma_{\alpha}^{p_{2}}\left(\overline{j_{2}}, \overline{n_{2}}\right)\right) \geq \alpha / 2 .
$$

We can now state Proposition 3.5 (similar to [6, Proposition 2.8]), which follows from the above lemmas.

Proposition 3.5. Assume that (H1), (H2), and (C) are satisfied. Let $\left(x_{n}\right) \subset H^{1,2}$ $(\mathbb{R}, T M)$ with $I\left(x_{n}\right) \rightarrow c$, $\left\|I^{\prime}\left(x_{n}\right)\right\|_{L^{2}} \rightarrow 0$, and $\left\|x_{n}-x_{n+1}\right\|_{L^{2}} \rightarrow 0$. Then there is a connected component $\Gamma$ of $\mathscr{C}$, with $I \equiv c$ on $\Gamma$, and $\operatorname{dist}_{H^{1,2}}\left(x_{n}, \Gamma\right) \rightarrow 0$ as $n \rightarrow \infty$. 
Proof. The proof of this proposition is analogous to that of [23, Lemma 4]. Note that by Lemma 3.3, for $n$ large, $x_{n} \in V_{\alpha}(\varepsilon)$. Since $\left\|x_{n}-x_{n+1}\right\|_{L^{2}} \rightarrow 0$, Lemma 3.4 implies that for $n$ large, $x_{n}$ stays in a fixed $U_{\alpha}^{p}(\bar{j}, \bar{u}, \varepsilon)$. But on $U_{\alpha}^{p}(\bar{j}, \bar{u}, \varepsilon)$, the Palais-Smale condition holds; hence $\left(x_{n}\right)$ is precompact. Its limit set is compact and connected since $\left\|x_{n}-x_{n+1}\right\|_{L^{2}} \rightarrow 0$; hence, it is contained in a connected component $\Gamma$ of $\mathscr{C} . I \equiv c$ on $\Gamma$ follows from Lemma 3.1(ii).

Proposition 3.5 has the following consequence, analogous to Lemma 3.2 in [6].

Corollary 3.1. Assume that (H1), (H2), and (C) are satisfied. Let $u \in C^{\infty}\left(\mathbb{R}^{2}\right.$, TM) be such that

$$
\bar{\partial} u+H^{\prime}(t, u)=0 \quad \text { and } \quad \int_{\mathbb{R}^{2}}\left|\partial_{s} u\right|^{2} d s d t<\infty .
$$

Assume, moreover, that $u(s, \cdot) \in H^{1,2}(\mathbb{R}, T M)$ for all $s$.

Let us define $I^{+}(u)=\lim _{s \rightarrow+\infty} I(u(s))$. There is a connected component $\Gamma_{+}$of $\mathscr{b}$ such that

$$
\operatorname{dist}_{L^{2}(\mathbb{R})}\left(u(s), \Gamma_{+}\right) \underset{s \rightarrow+\infty}{\longrightarrow} 0 \text { and } I\left(\Gamma_{+}\right)=I^{+}(u) .
$$

The same is true when one replaces $+\infty$ by $-\infty$.

4. An a priori estimate for the elements of $X_{R}$. In this part, we give a complement to the a priori estimate of [6, Proposition 3.9].

Lemma 4.1. Assume that (H1), (H2) are satisfied. Then

$$
\|x\|_{1,2}^{2} \leq K\left(\left\|I^{\prime}(x)\right\|_{L^{2}}^{2}+I(x)\right) \text { for all } x \in H^{1,2}(\mathbb{R}, T M),
$$

with a constant $K$ depending only on $a, c_{1}$, and $c_{2}$.

Proof. The proof is identical to that of Lemma 2.2 in [6].

Lemma 4.2. Assume that (H1), (H2), are satisfied.

(i) Ifthe image of $x \in H^{1,2}$ is contained in the zero section ofTM, then

$$
I(x) \geq 0
$$

The same is true for $x \in \mathscr{C}$.

(ii) For all $x=(q, p)$ in $H^{1,2}(\mathbb{R}, T M)$, we have

$$
I(x) \leq d_{1}\|\dot{q}\|_{L^{2}}^{2}+d_{2}
$$

with constants $d_{1}, d_{2}$ depending only on $b_{1}, b_{2}$.

Proof. See the discussion before Lemma 3.3 in [6]. 
Now let us introduce a further hypothesis on $H$.

(H3) In geodesic normal coordinates around each $x=(q, p) \in T M$, we have

$$
\left|\frac{\partial^{2} H}{\partial p^{2}}(t, x)\right|+\left|\frac{\partial^{2} H}{\partial p \partial q}(t, x)\right| \leq \text { const. }
$$

At the end of Section 5, we show how to remove this hypothesis.

For a subset $K \in \Omega_{T}^{\infty}$ and $\bar{n}=\left(n_{1}, \ldots, n_{p}\right) \in \mathbf{Z}^{p}, n_{i+1}-n_{i} \geq 2 T$, we can glue together curves in $K$ to get a subset $\bar{n} * K \subset \bar{n} * \Omega_{T}^{\infty}$. Set

$$
X_{\bar{n} * K, R}:=\bigcup_{\bar{q} \in \bar{n} * K} X_{\bar{q}, R},
$$

where $X_{\bar{q}, R}$ is the set defined in Section 2 .

Proposition 4.3. Assume that (H1), (H2), (H3), and (C) are satisfied. Let $\mathrm{K}$ be a compact subset of $\Omega_{T}^{\infty}$.

There are $\varepsilon_{0}>0$ and a family of positive constants $\left(c_{\alpha}, \rho_{\alpha}\right)_{\alpha \in \mathbb{N}^{2}}$, such that, if $\bar{n}=\left(n_{1}, \ldots, n_{p}\right) \in \mathbf{Z}^{p}$ satisfies $n_{i+1}-n_{i} \geq 2$ for all $i$, and $u \in X_{\bar{n} * K, R}$ satisfies

$$
\left|\partial^{\alpha} u(s, t)\right| \leq \varepsilon_{0}
$$

whenever

$$
\operatorname{dist}\left(t,\left\{\frac{n_{i}+n_{i+1}}{2}\right\}_{1 \leq i \leq p-1}\right) \leq 1
$$

and $|\alpha| \leq 2$, then

$$
\left|\partial^{\alpha} u(s, t)\right| \leq c_{\alpha} e^{-\rho_{\alpha} \operatorname{dist}\left(t,\left\{n_{i}\right\}_{1 \leq i \leq p}\right)}
$$

for any $\alpha \in \mathbb{N}^{2}$. These estimates depend neither on $R$ nor on $p$.

Proof. To prove Proposition 4.3, we use a cutoff function $\theta \in C^{\infty}(\mathbb{R},[0,1])$ such that $\theta(t)+\theta(-t)=1$, and

$$
\begin{cases}\theta \equiv 0 & \text { on }(-\infty,-1] \\ \theta \equiv 1 & \text { on }[1,+\infty)\end{cases}
$$

Define

$$
\left\{\begin{array}{l}
\Theta_{1}(t)=\theta\left(\frac{n_{1}+n_{2}}{2}-t\right), \\
\Theta_{i}(t)=\theta\left(t-\frac{n_{i-1}+n_{i}}{2}\right) \theta\left(\frac{n_{i+1}+n_{i}}{2}-t\right), \quad 2 \leq i \leq p-1, \\
\Theta_{p}(t)=\theta\left(t-\frac{n_{p}+n_{p-1}}{2}\right),
\end{array}\right.
$$

and $u_{i}(s, t)=\Theta_{i}(t) u(s, t), 1 \leq i \leq p$. This product makes sense if $\varepsilon_{0}$ is smaller than the injectivity radius of $M$. When $\Theta_{i}(t)=0$, take $u_{i}(s, t)=x_{0}$; when $\Theta_{i}(t)=1$, take 
$u_{i}(s, t)=u(t)$; when $0<\Theta_{i}(t)<1$, we have $\left|u(s, t)-x_{0}\right| \leq \varepsilon_{0}$, so we can define $u_{i}(s, t)=\exp _{x_{0}}\left[\Theta_{i}(t) \exp _{x_{0}}^{-1}(u(s, t))\right]$. One easily checks the following properties of $u_{i}$ :

$$
\begin{cases}u_{i}=0 & \text { outside }\left[\frac{n_{i-1}+n_{i}}{2}-1, \frac{n_{i+1}+n_{i}}{2}+1\right], \\ \bar{\partial} u_{i}+H^{\prime}\left(t, u_{i}\right) \equiv 0 & \text { on }\left[\frac{n_{i-1}+n_{i}}{2}+1, \frac{n_{i+1}+n_{i}}{2}-1\right], \\ \left|\partial^{\alpha}\left(\bar{\partial} u_{i}+H^{\prime}\left(t, u_{i}\right)\right)\right| \leq C \varepsilon_{0} & \text { everywhere, }|\alpha| \leq 2, \\ \tau u_{i}(R, t)=q_{i}\left(t-n_{i}\right), & \text { where } M_{0} \text { is the zero section of } M . \\ u_{i}(-R, t) \in M_{0}, & \end{cases}
$$

So we are led to use the following result.

Lemma 4.4. Assume that (H1), (H2), (H3), and (C) are satisfied. Let $K$ be a compact subset of $\Omega_{T}^{\infty}$, and take $r>0$.

There are constants $B>0, \delta(r)>0$, and $D(r)>0$ such that, for any $R \geq 1$, if $u \in C^{\infty} \cap H^{2,2}\left(Z_{R}, T M\right)$ satisfies

(i) $\tau u(R, \cdot)=q \in K, u(-R, t) \in M_{0}$,

(ii) $\sup _{s \in[-R, R]}\left\|\bar{\partial} u+H^{\prime}(t, u)\right\|_{L^{2}(d t)}+\left\|\bar{\partial} u+H^{\prime}(t, u)\right\|_{C^{2}\left(Z_{R}\right)} \leq \delta(r)$,

then $|u(s, t)| \leq r$ when $|t| \geq D(r)$, and $\left|\partial^{\alpha} u(s, t)\right| \leq B$ everywhere, for $|\alpha| \leq 2$.

Before proving Lemma 4.4, let us explain why it implies Proposition 4.3. Let us take $r>0$. From (4.2), we see that if we choose $\varepsilon_{0}(r)$ small enough, each $u_{i}\left(\cdot+n_{i}\right)$ satisfies the hypotheses of Lemma 4.4. Hence

$$
\left|u_{i}\right| \leq B \quad \text { everywhere, }
$$

and $\left|u_{i}(t)\right| \leq r$ whenever $\left|t-n_{i}\right| \geq D(r)$. As a consequence, we have

$$
|u| \leq B \quad \text { everywhere, }
$$

and $|u| \leq r$ when $\operatorname{dist}\left(t,\left\{n_{i}\right\}_{1 \leq i \leq p}\right) \geq D(r)$. Using a bubbling-off argument followed by a bootstrap as in [6], one can prove that

$$
\left|\partial^{\alpha} u\right| \leq B_{\alpha} \quad \text { for any } \alpha \in \mathbb{N}^{2},
$$

$B_{\alpha}$ depending only on $M$.

Then one uses the same arguments as in the proof of Lemmas 3.6 and 3.9 of [6]; when $r$ has been chosen small enough, one gets the estimates

$$
\left|\partial^{\alpha} u\right| \leq c_{\alpha} e^{-\rho_{\alpha} \operatorname{dist}\left(t,\left\{x_{i}\right\}_{1 \leq i \leq p}\right)},
$$

and Proposition 4.3 follows. 
ProofofLemma 4.4. Assumption (C) is only used in step 3 of this proof.

Step 1. Let $\delta$ be small, and let $u$ satisfy (i), (ii) of Lemma 4.4. If $s \leq s^{\prime}$, then

$$
I(u(s, \cdot)) \leq I\left(u\left(s^{\prime}, \cdot\right)\right)+\eta(\delta),
$$

and $\eta \rightarrow 0$ as $\delta \rightarrow 0$. Moreover, there is a finite constant $h$ such that for all $u$ and $\delta$ small enough,

$$
I(u(s, \cdot)) \leq h, \quad \forall s \in[-R, R]
$$

Proofofstep 1. We recall that $I(x) \geq 0$ for all $x \in \mathscr{C}$ (see Lemma 4.2).

Let us take $h=1+\sup \left\{I(x) \mid x \in H^{1,2}(\mathbb{R}, T M), \tau x \in K\right\}$. It follows from Lemma 4.2(ii) that $h<\infty$. We always have $h \geq 1+I(u(R, \cdot))$. We prove the inequality

$$
I(u(s, \cdot)) \leq I\left(u\left(s^{\prime}, \cdot\right)\right)+\eta(\delta),
$$

provided that $s \leq s^{\prime}$ and $I\left(u\left(s^{\prime}, \cdot\right)\right) \leq h-(1 / 2)$. Then taking $s^{\prime}=R$ and $\eta(\delta) \leq 1 / 2$, it follows that $I(u(s, \cdot)) \leq h-(1 / 2)$ is satisfied for any $s \leq R$, thus the condition $I\left(u\left(s^{\prime}, \cdot\right)\right) \leq h-(1 / 2)$ will always be satisfied, and step 1 will follow.

If $I\left(u\left(s^{\prime}, \cdot\right)\right) \leq h-(1 / 2)$, we may associate, to any $1 / 2>\eta>0$, the quantity $\zeta(h, \eta)$ defined in Lemma 3.1. Take $J=\left[I\left(u\left(s^{\prime}, \cdot\right)\right), I\left(u\left(s^{\prime}, \cdot\right)\right)+\eta\right]$ and $J^{\prime} \subset J \cap$ $(\mathbb{R} \backslash F),\left|J^{\prime}\right|=\zeta(h, \eta)$.

From Lemma 3.3, there is a positive constant $\delta>0$ such that

$$
\left\|I^{\prime}(x)\right\|_{L^{2}} \leq \delta \Longrightarrow I(x) \notin J^{\prime \prime},
$$

where $J^{\prime \prime}$ is an interval with the same center as $J^{\prime}$, and $\left|J^{\prime \prime}\right|=\left|J^{\prime}\right| / 2$.

Now, assume that $I(u(s, \cdot)) \geq I\left(u\left(s^{\prime}, \cdot\right)\right)+\eta$. Then, for some $s^{\prime \prime} \in\left[s, s^{\prime}\right]$, we have

$$
I\left(u\left(s^{\prime \prime}, \cdot\right)\right) \in J^{\prime \prime} \quad \text { and }\left.\quad \frac{d}{d s} I(u(s, \cdot))\right|_{s=s^{\prime \prime}} \leq 0 .
$$

Using

$$
\left\|\partial_{s} u\left(s^{\prime \prime}, \cdot\right)-I^{\prime}\left(u\left(s^{\prime \prime}, \cdot\right)\right)\right\|_{L^{2}} \leq \delta,
$$

we obtain

$$
\begin{aligned}
\delta^{2} & \geq\left\|u_{s}\left(s^{\prime \prime}\right)\right\|_{L^{2}}^{2}-2<u_{s}\left(s^{\prime \prime}\right), I^{\prime}\left(u\left(s^{\prime \prime}\right)\right)>_{L^{2}}+\left\|I^{\prime}\left(u\left(s^{\prime \prime}\right)\right)\right\|_{L^{2}}^{2} \\
& \geq\left\|I^{\prime}\left(u\left(s^{\prime \prime}\right)\right)\right\|_{L^{2}}^{2},
\end{aligned}
$$

hence $I\left(u\left(s^{\prime \prime}\right)\right) \notin J^{\prime \prime}$, and we have a contradiction.

So we have proved that

$$
I(u(s, \cdot)) \leq I\left(u\left(s^{\prime}, \cdot\right)\right)+\eta \quad \text { for } \delta \text { small enough. }
$$

Step 2. There is a constant $B$ such that

$$
\left|\partial^{\alpha} u\right| \leq B \quad \text { for }|\alpha| \leq 2 .
$$


Proofofstep 2. Take $\sigma>0$. There is a constant $C(\sigma)$ such that, for any interval $I \subset[-R, R]$ of size $\sigma$, one can find $s_{I} \in I$ such that $\left\|u\left(s_{I}, \cdot\right)\right\|_{H^{1,2}(d t)} \leq C$. Otherwise, by Lemma 4.1, we would find intervals $I \subset[-R, R]$ of size $\sigma$ with $\inf _{s \in I}(I+$ $\left.\left\|I^{\prime}\right\|_{L^{2}}^{2}\right)$ arbitrarily large. We see from step 1 that this is impossible, since $I(u(R))-$ $I(u(-R)) \leq h$.

So we can cut $[-R, R]$ in a finite partition of intervals $\left(I_{\lambda}\right)_{\lambda \in \Lambda}$ such that $\left|I_{\lambda}\right| \leq 2 \sigma$ for any $\lambda \in \Lambda$, and $\|u(s, \cdot)\|_{H^{1,2}(d t)} \leq C(\sigma)$ for any boundary point $s$ of $I_{\lambda}$, except possibly for zero and $R$.

The inclusion $H^{1,2}(\mathbb{R}) \subset C^{0}(\mathbb{R})$ yields uniform estimates for $u$ on the boundary of $I_{\lambda}$. The $C^{2}$-estimate on $\bar{\partial} u+H^{\prime}(t, u)$ and assumption (H3) allow us to apply the maximum principle as in [6, proof of Lemma 3.3] to obtain the uniform estimate

$$
|u(s, t)| \leq B_{*} \quad \text { for all }(s, t) \in Z_{R} .
$$

This has been the only step where we used (H3).

From

$$
\begin{aligned}
h \geq I\left(u\left(s_{2}\right)\right)-I\left(u\left(s_{1}\right)\right) & =\int_{s_{1}}^{s_{2}}\left\langle u_{s}, I^{\prime}(u)\right\rangle_{L^{2}} d s \\
& \geq \int_{s_{1}}^{s_{2}}\left\|u_{s}\right\|_{L^{2}}^{2} d s-\delta \int_{s_{1}}^{s_{2}}\left\|u_{s}\right\|_{L^{2}} d s \\
& \geq \int_{s_{1}}^{s_{2}}\left\|u_{s}\right\|_{L^{2}}^{2} d s-\delta \sqrt{s_{2}-s_{1}}\left(\int_{s_{1}}^{s_{2}}\left\|u_{s}\right\|_{L^{2}}^{2} d s\right)^{1 / 2},
\end{aligned}
$$

we obtain

$$
\int_{s_{1}}^{s_{2}}\left\|u_{s}\right\|_{L^{2}}^{2} d s \leq c\left(h+\delta^{2}\left(s_{2}-s_{1}\right)\right) .
$$

So $\int_{s_{1}}^{s_{2}}\left\|u_{s}\right\|_{L^{2}(d t)}^{2} d s$ is bounded for finite intervals [s,$\left.s_{2}\right]$. Using this, a classical bubbling-off analysis for $|\alpha|=1$ (see, e.g., [5, Chapter 5]), followed by a bootstrap to reach $|\alpha|=2$, gives the final estimate

$$
\left|\partial^{\alpha} u(s, t)\right| \leq B \quad \text { for any }|\alpha| \leq 2 .
$$

Note that $u$ is even bounded in $C^{2, \gamma}$ for any $0<\gamma<1$ by the bootstrap argument.

Step 3. We use the assumption (C) in this step. Given $\xi, \beta>0$, for $\delta$ small enough, there is a partition of $[-R, R]$ by $N$ intervals $I_{0}, \ldots, I_{N}, N \leq \mathcal{N}(\xi, \beta)$ even, with $I_{0}=\left[-R, S_{1}\right], I_{k}=\left[S_{k}, S_{k+1}\right]$ for $1 \leq k \leq N-1, I_{N}=\left[S_{N}, R\right]$, and such that the following hold.

(a) There is an $L(\xi, \beta)$ such that, for $k=2 q$,

$$
0<S_{2 q+1}-S_{2 q}<L(\xi, \beta) .
$$

(b) For $k=2 q+1$, there are $1 \leq p \leq h / \rho, \bar{j} \in\left(J_{\beta}\right)^{p}, \bar{n} \in Z^{p}(\bar{j}, \xi)$ such that

$$
\operatorname{dist}_{L^{2}(\mathbb{R})}\left(u(s, \cdot), \Gamma_{\beta}^{p}(\bar{j}, \bar{n})\right) \leq \xi \quad \text { for any } s \in I_{k} .
$$


Here, $h$ is the constant of step 1 , and $\rho, J_{\beta}$, and $\Gamma_{\beta}^{p}(\bar{j}, \bar{n})$ were defined in the discussion preceding Lemma 3.3.

Proofofstep 3. The proof is very similar to the proof of the first step in [6, Lemma 3.8]. Given $L>0$, in each interval $[m(L / 2),(m+1) L / 2]$, with $-(2 R / L) \leq m \leq$ $(2 R / L)-1$, there is $s_{m}$ such that

$$
\left\|I^{\prime}\left(u\left(s_{m}\right)\right)\right\|_{L^{2}(d t)}^{2} \leq \frac{2 h}{L} .
$$

So, from Lemma 3.3, given $\varepsilon, \alpha>0$, there are $L_{\varepsilon, \alpha}>0$ and a finite sequence $\left(s_{m}\right)$ such that $0<s_{m+1}-s_{m}<L$ and $u\left(s_{m}, \cdot\right) \in V_{\alpha}(\varepsilon)$ for all $m$.

Case 1. $u\left(s_{m}, \cdot\right), u\left(s_{m+1}, \cdot\right)$ are not in the same $U_{\alpha}^{p_{*}}\left(\overline{j_{*}}, \overline{n_{*}}, \varepsilon\right)$. In this case, let $\left[s_{m}^{\prime}, s_{m+1}^{\prime}\right] \subset\left[s_{m}, s_{m+1}\right]$ be an interval such that $u\left(s_{m}^{\prime}\right)$ and $u\left(s_{m+1}^{\prime}\right)$ belong to different $U_{\alpha}^{p_{*}}\left(\overline{j_{*}}\right.$,

$\left.\overline{n_{*}}, \varepsilon\right)$ and such that $u\left(\left(s_{m}^{\prime}, s_{m+1}^{\prime}\right)\right)$ does not meet $V_{\alpha}(\varepsilon)$. By Lemma 3.3, the latter condition implies, for $\alpha \leq \alpha(h)$ sufficiently small,

$$
\left\|I^{\prime}(u(s))\right\|_{L^{2}} \geq \mu \quad \text { for all } s \in\left[s_{m}^{\prime}, s_{m+1}^{\prime}\right] .
$$

From Lemma 3.4, we obtain, for $\alpha \leq \alpha(h)$ and $\varepsilon \leq \varepsilon(\alpha)$ small enough,

$$
\left\|u\left(s_{m+1}^{\prime}\right)-u\left(s_{m}^{\prime}\right)\right\|_{L^{2}} \geq \frac{\alpha}{4} .
$$

Combining these, we estimate

$$
\begin{aligned}
& I\left(u\left(s_{m+1}^{\prime}\right)\right)-I\left(u\left(s_{m}^{\prime}\right)\right) \\
& \quad=\int_{s_{m}^{\prime}}^{s_{m+1}^{\prime}}<\partial_{s} u(s), I^{\prime}(u(s))>_{L^{2}} d s \\
& \quad=\int_{s_{m}^{\prime}}^{s_{m+1}^{\prime}}\left(\frac{1}{2}\left\|\partial_{s} u(s)\right\|_{L^{2}}^{2}+\frac{1}{2}\left\|I^{\prime}(u(s))\right\|_{L^{2}}^{2}-\frac{1}{2}\left\|\bar{\partial} u+H^{\prime}(t, u)\right\|_{L^{2}}^{2}\right) d s \\
& \quad \geq \int_{s_{m}^{\prime}}^{s_{m+1}^{\prime}}\left(\left\|\partial_{s} u(s)\right\|_{L^{2}}\left\|I^{\prime}(u(s))\right\|_{L^{2}}-\frac{1}{2}\left\|\bar{\partial} u+H^{\prime}(t, u)\right\|_{L^{2}}^{2}\right) d s \\
& \quad \geq \mu \int_{s_{m}^{\prime}}^{s_{m+1}^{\prime}}\left\|\partial_{s} u(s)\right\|_{L^{2}} d s-\frac{L}{2} \delta^{2} \\
& \geq \mu\left\|u\left(s_{m+1}^{\prime}\right)-u\left(s_{m}^{\prime}\right)\right\|_{L^{2}}-\frac{L}{2} \delta^{2} \geq \frac{\mu \alpha}{4}-\frac{L}{2} \delta^{2} \geq \frac{\mu \alpha}{8}
\end{aligned}
$$

for $\delta \leq \delta(\alpha)$ small enough. On the other hand, we know from step 1 that

$$
\begin{aligned}
& I\left(u\left(s_{m}\right)\right) \leq I\left(u\left(s_{m}^{\prime}\right)\right)+\eta(\delta), \\
& I\left(u\left(s_{m+1}\right)\right) \geq I\left(u\left(s_{m+1}^{\prime}\right)\right)-\eta(\delta) .
\end{aligned}
$$


Hence for $\delta \leq \delta(\alpha)$ sufficiently small, we get

$$
I\left(u\left(s_{m+1}\right)\right)-I\left(u\left(s_{m}\right)\right) \geq \chi(\alpha)>0
$$

with some constant $\chi(\alpha)$.

So for each interval $\left[s_{m}, s_{m+1}\right]$ with $u\left(s_{m}, \cdot\right), u\left(s_{m+1}, \cdot\right)$ not lying in the same $U_{\alpha}^{p_{*}}\left(\overline{j_{*}}, \overline{n_{*}}, \varepsilon\right)$, the action increases by at least $\chi(\alpha)$. Again by step 1 , between two such intervals the action can only decrease by $\eta(\delta)<\chi(\alpha) / 2$ if $\delta \leq \delta(\alpha)$ is small enough. Hence

$$
\operatorname{Card}\left\{m \mid u\left(s_{m}, \cdot\right), u\left(s_{m+1}, \cdot\right) \text { are not in the same } U_{\alpha}^{p_{*}}\left(\overline{j_{*}}, \overline{n_{*}}, \varepsilon\right)\right\} \leq \frac{2 h}{\chi(\alpha)} .
$$

Case 2. $u\left(s_{m}, \cdot\right), u\left(s_{m+1}, \cdot\right)$ are in the same $U_{\alpha}^{p_{*}}\left(\overline{j_{*}}, \overline{n_{*}}, \varepsilon\right)$. We now fix $\beta>0$ small. For $\xi>0$ small enough, we are going to choose $\alpha$ "much smaller" than $\beta, \varepsilon$ "much smaller" than $\xi$, and $\delta$ very small, to prove step 3 . Let us explain this.

We define

$$
\mu(\xi, \beta)=\inf \left\{\left\|I^{\prime}(x)\right\|_{L^{2}} \mid \operatorname{dist}_{L^{2}}\left(x, G_{\beta}(\xi)\right) \in\left[\frac{\xi}{2}, \xi\right]\right\} .
$$

From Lemma 3.3, we know that $\mu>0$.

Given $\beta$ and $\xi$, if $\varepsilon$ is small enough, we can choose $1 \leq p \leq h / \rho, \bar{j} \in\left(J_{\beta}\right)^{p}, \bar{n} \in$ $Z^{p}(\bar{j}, \xi)$, such that

$$
u\left(s_{m}\right) \in U_{\beta}^{p}(\bar{j}, \bar{n}, \xi / 2) .
$$

Assume by contradiction that there exists $s^{*} \in\left[s_{m}, s_{m+1}\right]$ minimal, such that

$$
\operatorname{dist}_{L^{2}}\left(u(s, \cdot), \Gamma_{\beta}^{p}(\bar{j}, \bar{n})\right)=\xi .
$$

Then, from Lemma 3.4, for a given $\beta$, if $\xi$ is small enough,

$$
\operatorname{dist}_{L^{2}}\left(u(s, \cdot), \Gamma_{\beta}^{p^{\prime}}\left(\overline{j^{\prime}}, \overline{n^{\prime}}\right)\right) \geq \xi
$$

for all $p^{\prime} \geq 1$ and $\overline{j^{\prime}} \in\left(J_{\beta}\right)^{p^{\prime}}, \overline{n^{\prime}} \in Z^{p^{\prime}}\left(\overline{j^{\prime}}, \xi\right)$.

So there is $s_{*}$ in $\left[s_{m}, s^{*}\right]$, such that

$$
\operatorname{dist}_{L^{2}}\left(u\left(s_{*}\right), G_{\beta}(\xi)\right)=\xi / 2, \quad \operatorname{dist}_{L^{2}}\left(u\left(s^{*}\right), G_{\beta}(\xi)\right)=\xi ;
$$

and for any $s \in\left[s_{*}, s^{*}\right]$,

$$
\operatorname{dist}_{L^{2}}\left(u(s), G_{\beta}(\xi)\right) \in\left[\frac{\xi}{2}, \xi\right] .
$$

As a consequence, if we impose $\delta \leq \mu / 2$,

$$
I\left(u\left(s^{*}\right)\right)-I\left(u\left(s_{*}\right)\right)=\int_{s_{*}}^{s^{*}}\left\langle\partial_{s} u, I^{\prime}(u)\right\rangle \geq \frac{\xi}{4} \mu(\xi, \beta) .
$$


So step 1 implies that $I\left(u\left(s^{*}\right)\right)-I\left(u\left(s_{*}\right)\right) \geq(\xi / 4) \mu(\xi, \beta)-2 \eta(\delta)$. But, since $u\left(s_{m}, \cdot\right)$ and $u\left(s_{m+1}, \cdot\right)$ are in the same $U_{\alpha}^{p_{*}}\left(\overline{j_{*}}, \overline{n_{*}}, \varepsilon\right)$, Lemma 3.1(iii) implies that $I\left(u\left(s^{*}\right)\right)-$ $I\left(u\left(s_{*}\right)\right)$ is arbitrarily small for $\alpha, \varepsilon$ arbitrarily small. This gives a contradiction for $\delta, \alpha$, and $\varepsilon$ very small.

We have thus proved, by contradiction, that given $\beta$ and $\xi$ small enough, for $\varepsilon, \alpha$, and $\delta$, very small, we have

$$
\operatorname{dist}_{L^{2}}\left(u(s, \cdot), \Gamma_{\beta}^{p}(\bar{j}, \bar{n})\right)<\xi, \quad \forall s \in\left[s_{m}, s_{m+1}\right],
$$

for some $1 \leq p \leq A / \rho, j \in\left(J_{\beta}\right)^{p}, \bar{n} \in Z^{p}(\bar{j}, \xi)$.

The existence of the partition $I_{0}, \ldots, I_{N}$ easily follows from the above arguments, with a bound on $N$ coming from case 1 .

Step 4. We fix $\beta$ and $\xi$ small, such that

$$
\left\|x-x_{0}\right\|_{\infty} \geq 2 \xi \text { for all } x \in \mathscr{b} .
$$

Let us now take an arbitrary $r<\xi$. We find, by induction on $q$, two functions $\delta_{q}(r)$ and $D_{q}(r)$ such that

$$
\|u\|_{C^{0}\left(I_{N-q} \times\left(\mathbb{R} \backslash\left[-D_{q}, D_{q}\right]\right)\right)} \leq r
$$

when $\delta \leq \delta_{q}(r)$. This is done using the estimates of steps 1 and 2 and arguments similar to those in [6, proof of Lemma 3.8, second step].

Since $0 \leq q \leq \mathcal{N}(\xi, \beta)$, we may replace $\delta_{q}$ by $\delta=\min _{q} \delta_{q}$ and $D_{q}$ by $D=$ $\max _{q} D_{q}$, and get the estimate

$$
\|u\|_{C^{0}([0, T] \times \mathbb{R} \backslash[-D(r), D(r)])} \leq r
$$

when $\delta \leq \delta(r)$. Thus Lemma 4.4 is proved.

5. Existence of solutions with $p$ bumps. Let $T \geq 1$ and $\Omega_{T}:=\left\{q \in C^{0}(\mathbb{R}, M) \mid\right.$ $q(t)=q_{0}$ for $\left.|t| \geq T-1\right\}$. Let $K \subset \Omega_{T}$ be a connected compact finite-dimensional submanifold with boundary containing the constant loop $q_{0}$. Choose $p \in \mathbb{N}$ and $n=\left(n_{1}, \ldots, n_{p}\right) \in \mathbf{Z}^{p}$ with $n_{i+1}-n_{i} \geq D$. Here $D \geq 2 T$ is taken large enough to have

$$
c_{\alpha} e^{-\rho_{\alpha}((D / 2)-1)}<\varepsilon_{0}, \quad \forall|\alpha| \leq 2,
$$

where $\varepsilon_{0}, c_{\alpha}$, and $\rho_{\alpha}$ are from Proposition 4.3.

Define

$$
n * \Omega_{T}:=\left\{q(t)=\sum_{i=1}^{p} q_{i}\left(t-n_{i}\right) \mid q_{i} \in \Omega_{T}\right\}
$$

(where the meaning of the sum is clear in view of the compact supports). In [6], we introduced a smoothing operator

$$
\mathscr{g}: \Omega_{T} \rightarrow \Omega_{T}^{\infty}
$$


$\mathscr{F}$ induces a smoothing operator $\mathscr{F}: n * \Omega_{T} \rightarrow n * \Omega_{T}^{\infty}$ sending $n * K$ to the compact set $n * \tilde{K}$.

Following [6], we define the following for $\bar{q} \in n * \Omega_{T}^{\infty}$ and $R>1$ :

$$
\begin{aligned}
\mathscr{E}_{\bar{q}, R}:=\left\{u \in H^{4,2}([-R, R] \times \mathbb{R}, T M) \mid u(-R, t)\right. & \in M, \\
u(R, t) & \left.\in T_{\bar{q}(t)} M \text { for all } t \in \mathbb{R}\right\}
\end{aligned}
$$

and

$$
\begin{aligned}
X_{R}=X_{n * K, R}= & \left\{u \in \bigcup_{q \in n * K} \mathscr{E}_{\mathcal{E} q, R} \mid \bar{\partial} u+H^{\prime}(t, u)=0,\right. \\
& \left.\left|\partial^{\alpha} u(s, t)\right| \leq \varepsilon_{0} \text { for }\left|t-\frac{n_{i}+n_{i+1}}{2}\right| \leq 1,1 \leq i<p,|\alpha| \leq 2\right\} .
\end{aligned}
$$

As in [6], we define a Hilbert manifold

$$
\mathscr{E}:=\left\{u: \mathbb{R}^{2} \rightarrow T M \mid\|u\|_{H_{\text {semi-loc }}^{4,2}}<\infty\right\},
$$

where

$$
\|u\|_{H_{\mathrm{semi}-\mathrm{loc}}^{4,2}}^{2}=\sum_{p \geq 1} \frac{1}{2^{p}}\|u\|_{H^{4,2}([-p, p] \times \mathbb{R}, T M)}^{2} .
$$

Note that in the definitions of $\mathscr{E}_{\bar{q}}, R$ and $\mathscr{E}$, we use the $H^{4,2}$ Sobolev norms instead of the $H^{2,2}$ norms used in [6]. The reason is that we want the elements of our Hilbert manifolds to be of class $C^{2}$. Of course, this does not make any difference for the elements of $X_{R}$, since they are smooth with exponential decay of all derivatives, as $|t|$ goes to infinity.

Taking maps $\phi_{R} \in C^{\infty}(\mathbb{R},[-R, R])$ such that $\phi_{R}(s)=s$ for $|s| \leq R-1, \phi_{R}(s) \equiv$ $-R$ for $s \leq-R$, and $\phi_{R}(s) \equiv R$ for $s \geq R$, we obtain embeddings

$$
\begin{aligned}
P_{R}: X_{R} & \rightarrow \mathscr{E} \\
u & \mapsto P_{R} u(s, t):=u\left(\phi_{R}(s), t\right) .
\end{aligned}
$$

Let

$$
\begin{array}{r}
X_{\infty}:=X_{n * K, \infty}:=\left\{u \in \mathscr{E} \mid \text { there exist sequences } R_{j} \rightarrow \infty, u_{j} \in X_{n * K, R_{j}}\right. \\
\text { such that } \left.P_{R_{j}}\left(u_{j}\right) \rightarrow u \text { in } \mathscr{E}\right\} .
\end{array}
$$

We have a natural projection

$$
\begin{aligned}
\pi: \mathscr{E} & \rightarrow \Omega=\left\{q \in C^{0}(\mathbb{R}, M) \mid q(t) \rightarrow q_{0} \text { as } t \rightarrow \pm \infty\right\} \\
& u \mapsto \tau \circ u(0, \cdot) .
\end{aligned}
$$

The definition of $X_{\infty}$ as the limits of sequences in $X_{R_{j}}$ implies that $\pi\left(X_{\infty}\right)$ is actually contained in 


$$
\tilde{\Omega}_{n}=\left\{q \in \Omega|| q(t)-q_{0} \mid \leq \varepsilon_{0} \text { for dist }\left(t,\left\{\frac{n_{i}+n_{i+1}}{2}\right\}_{1 \leq i<p}\right) \leq 1\right\} .
$$

Since $n * \Omega_{T}$ is a deformation retract of $\tilde{\Omega}_{n}$, the injection $j: n * \Omega_{T} \hookrightarrow \tilde{\Omega}_{n}$ induces an isomorphism $j^{*}$ in cohomology. Therefore an $\tilde{\alpha} \in \bar{H}^{*}\left(\tilde{\Omega}_{n}\right)$ corresponds to each $\alpha \in \bar{H}^{*}\left(n * \Omega_{T}\right)$. Here we denote the Alexander-Spanier cohomology by $\bar{H}^{*}$.

Now we are ready to state the main result of this section.

Proposition 5.1. Assume that (H1), (H2), (H3), and (C) are satisfied. Let $\alpha \in$ $\bar{H}^{*}\left(n * \Omega_{T}, \mathbf{Z}_{2}\right)$ satisfy $0 \neq i_{K}^{*} \alpha \in \bar{H}^{*}\left(n * K, \mathbf{Z}_{2}\right)$, where $i_{K}=n * K \hookrightarrow n * \Omega_{T}$ denotes the inclusion. Then

$$
0 \neq\left(\left.\pi\right|_{X_{n * K, \infty}}\right)^{*} \tilde{\alpha} \in \bar{H}^{*}\left(X_{n * K, \infty}, \mathbf{Z}_{2}\right) .
$$

Proof. The proof is a modification of the proof of Proposition 4.7 in [6], and it relies crucially on Proposition 4.3 of the preceding section.

We start with some definitions. For $\bar{q} \in n * \Omega_{T}^{\infty}$, let

$$
\begin{aligned}
U_{\bar{q}, R}:=\left\{u \in \mathscr{C}_{\bar{q}, R}:\left|\partial^{\alpha} u(s, t)\right|\right. & <\varepsilon_{0} \\
& \text { for } \left.\operatorname{dist}\left(t,\left\{\frac{n_{i}+n_{i+1}}{2}\right\}_{1 \leq i<p}\right) \leq 1,|\alpha| \leq 2\right\} .
\end{aligned}
$$

$U_{\bar{q}, R}$ is an open subset of $\mathscr{C}_{\bar{q}, R}$ for the $H^{4,2}\left(Z_{R}, T M\right)$-topology.

Define

$$
\mathscr{E}_{R}:=\mathscr{E}_{n * \Omega_{T}, R}:=\left\{(q, u) \mid q \in n * \Omega_{T}, u \in \mathscr{E}_{\mathscr{q} q, R}\right\}
$$

and

$$
u_{R}:=U_{n * \Omega_{T}, R}:=\left\{(q, u) \mid q \in n * \Omega_{T}, u \in \mathcal{U}_{\mathscr{F q}, R}\right\} .
$$

$U_{R}$ is an open subset of $\mathscr{E}_{R}$.

The Cauchy-Riemann operator $(q, u) \mapsto \bar{\partial} u+H^{\prime}(t, u)$ defines a smooth section in the Hilbert bundle $\mathscr{F}$ over $\mathscr{E}_{R}$ with fibres $\mathscr{F}(q, u)=H^{3,2}([-R, R] \times \mathbb{R}, u * T T M)$. By Kuiper's theorem, this bundle is trivial, and we may regard the Cauchy-Riemann operator as a smooth map

$$
\bar{\partial}_{H}: \mathscr{E}_{R} \rightarrow F=H^{3,2}\left([-R, R] \times \mathbb{R}, \mathbb{C}^{n}\right) .
$$

We also regard the projection

$$
\begin{aligned}
\pi_{R}: \mathscr{E}_{R} & \rightarrow n * \Omega_{T} \\
(q, u) & \mapsto q
\end{aligned}
$$


66

and the combined operator

$$
f_{R}:=\pi_{R} \oplus \bar{\partial}_{H}: \mathscr{E}_{R} \rightarrow n * \Omega_{T} \times F
$$

Let

$$
A_{R}:=A_{n * K, R}:=\left(\pi_{R} \oplus \bar{\partial}_{H}\right)^{-1}(n * K \times\{0\})
$$

be the space of solutions of the Cauchy-Riemann equation with boundary conditions in $n * K$. Now we make the following crucial observation about $A_{R}$ :

$$
\text { For all } R>0, \quad A_{R} \cap \partial U_{R}=\emptyset \text {. }
$$

To see this, observe that for an element $(q, u) \in A_{R} \cap \bar{U}_{R}, u$ satisfies the hypothesis of Proposition 4.3. Hence $u$ has exponential decay $\left|\partial^{\alpha} u(s, t)\right| \leq c_{\alpha} e^{-\operatorname{dist}\left(t,\left\{n_{i}\right\}_{1 \leq i \leq p}\right)}$. For

$$
\operatorname{dist}\left(t,\left\{\frac{n_{i}+n_{i+1}}{2}\right\}_{1 \leq i<p}\right) \leq 1 \text {, }
$$

this implies

$$
\begin{aligned}
\left|\partial^{\alpha} u(s, t)\right| & \leq c_{\alpha} e^{-\rho_{\alpha}((D / 2)-1)} \\
& <\varepsilon_{0}
\end{aligned}
$$

in view of inequality $(5.1)$. Thus $(q, u)$ actually lies in the interior of $U_{R}$, and (5.2) is proved.

In [6, Proposition 3.7 and Corollary 4.4], we obtained the following properties:

(i) $A_{R}$ is compact for every $R$;

(ii) for $(q, u) \in A_{R}$, the linearization $D f_{R}(q, u)$ is a semi-Fredholm operator;

(iii) $D f_{R}\left(q_{0}, x_{0}\right)$ is an isomorphism;

(iv) $f_{R}^{-1}\left(\left(q_{0}, 0\right)\right)=\left\{\left(q_{0}, x_{0}\right)\right\}$.

In fact, we proved this for $D f: H^{2,2} \rightarrow H^{1,2}$, but there is no additional difficulty for Df $: H^{4,2} \rightarrow H^{3,2}$.

Now, from observation (5.2) and $\left(q_{0}, x_{0}\right) \in U_{R}$, it follows that the connected component $\tilde{A}_{R}$ of $A_{R}$ containing $\left(q_{0}, x_{0}\right)$ lies inside $U_{R}$. So we can argue as in the proof of Proposition 4.5 of [6], using properties (i)-(iii), to find an open connected neighborhood $\tilde{U}_{R} \subset U_{R}$ of $\tilde{A}_{R}$ such that $\partial \tilde{U}_{R} \cap A_{R}=\emptyset$, and $\left.f_{R}\right|_{\tilde{U}_{R}}$ is a proper Fredholm operator of index zero.

Property (iv) implies

$$
\operatorname{deg}\left(f_{R}, \tilde{U}_{R},(q, 0)\right)=1
$$

for all $q \in n * K$, where deg is the $\mathbf{Z}_{2}$ degree as defined in [25]. As in the proof of Lemma 4.6 of [6], it follows that

$$
\left(\left.\pi_{R}\right|_{\tilde{A}_{R}}\right)^{*} \alpha \neq 0
$$


Now $(q, u) \mapsto u$ yields a topological embedding of $\tilde{A}_{R}$ in $X_{R}=X_{n * K, R}$. Since the smoothing operator $\mathscr{g}$ is homotopic to the identity, we obtain

$$
\left(\pi_{R} \mid X_{R}\right)^{*} \alpha \neq 0
$$

where $\pi_{R}: X_{R} \rightarrow \tilde{\Omega}_{n}$ is the projection $u \mapsto \tau \circ u(R, \cdot)$. By construction, $X_{R}$ projects everywhere into $\tilde{\Omega}_{n}$, and $\pi_{R}$ is homotopic to $\pi_{0}: X_{R} \rightarrow \tilde{\Omega}_{n}, u \mapsto \tau \circ u(0, \cdot)$. Hence

$$
\left(\pi_{0} \mid X_{R}\right)^{*} \alpha \neq 0
$$

Finally, we let $R \rightarrow \infty$ and use tautness of the Alexander-Spanier cohomology, as in the proof of Proposition 4.7 in [6], to get

$$
\left(\pi \mid X_{\infty}\right)^{*} \alpha \neq 0
$$

Consider $I^{+}: \mathscr{E} \rightarrow \mathbb{R} \cup\{\infty\}$ defined by

$$
I^{+}(u)=\sup _{s \in \mathbb{R}} I(u(s)) .
$$

Recall the notation

$$
X=\left\{u \in \mathscr{E}: u_{s}+J(u) u_{t}+H^{\prime}(t, u)=0, \int_{\mathbb{R}^{2}}\left|u_{s}\right|^{2} d s d t<\infty\right\}
$$

for the set of infinite gradient lines of bounded energy.

For a nonzero $\alpha \in \bar{H}^{*}\left(\Omega, \mathbf{Z}_{2}\right)$, define the minimax value

$$
m(\alpha)=\inf _{\substack{A \subset X \\\left(\left.\pi\right|_{A}\right)^{*} \alpha \neq 0}}\left(\sup _{A} I^{+}\right) .
$$

We have $m(\alpha)<\infty$ for all $\alpha \neq 0$ (see [6, Proposition 4.7]). Fix a compact set $K \subset \Omega_{T}$ as before, with $i_{K}: K \rightarrow \Omega_{T}$ as the inclusion map.

Theorem 5.2. Assume that (H1), (H2), (H3), and (C) are satisfied. There exists a compact subset $\mathscr{C}_{0}$ of $\mathscr{C}$ (depending on $K$ ) such that for all $\epsilon>0$, we can find $N(\epsilon)>0$ with the following property:

Whenever $\alpha_{j} \in \bar{H}^{l_{j}}\left(\Omega, \mathbf{Z}_{2}\right), l_{j}>0, j=1, \ldots, p$, and $n=\left(n_{1}, \ldots, n_{p}\right) \in \mathbf{Z}^{p}$ satisfy

(a) $i_{K}^{*}\left(\alpha_{j}\right) \neq 0$ for all $j$ and

(b) $n_{j+1}-n_{j} \geq 2 N(\epsilon)$ for all $j$,

then there exist homoclinic orbits $y_{1}, \ldots, y_{p} \in \mathscr{C}_{0}$ and $y \in \mathscr{C}$, satisfying

(i) $\left|y_{j}(t)-x_{0}\right| \leq \epsilon$ for all $j$ and for $|t| \geq N(\epsilon)-1$,

(ii) $I\left(y_{j}\right) \geq m\left(\alpha_{j}\right)-\epsilon$ for all $j$, and

(iii) $\left|y(t)-\sum_{j=1}^{p} n_{j} * y_{j}\right| \leq \epsilon$ for all $t \in \mathbb{R}$. 
Proof. Regard $X_{K}=X_{0 * K, \infty}$. It follows from the definition of $X_{K}$ and hypothesis (H3) that

$$
I^{+}(u) \leq C
$$

for all $u \in X_{K}$, with a constant $C$ depending only on $K$ (see [6, discussion before Lemma 3.3]).

Proposition 3.9 of [6] implies that $X_{K}$ is precompact in $\mathscr{E}$. Hence $\pi\left(\bar{X}_{K}\right)$ is a compact subset of the loop space $\Omega$, from which we deduce that the pullback cohomology

$$
\left(\left.\pi\right|_{X_{K}}\right)^{*}\left(\bar{H}^{*}\left(\Omega, \mathbf{Z}_{2}\right)\right)
$$

is a finite-dimensional vector space over $\mathbf{Z}_{2}$ (see, for instance, [5]). By tautness of the Alexander-Spanier cohomology, there exists a neighborhood $\mathcal{U}$ of $X_{K}$ in $\mathscr{E}$, with canonical inclusion $j: X_{K} \rightarrow \mathcal{U}$, such that the following diagram commutes:

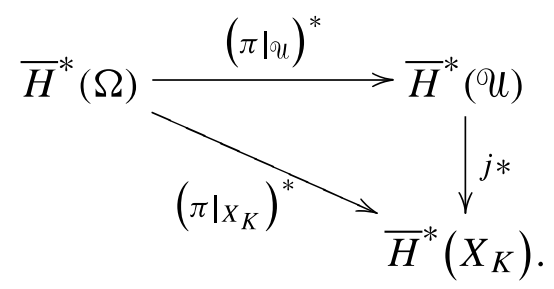

For $u \in X_{n * K, \infty}$ and $n \in \mathbf{Z}^{p}$, we have

$$
\left|u(s, t)-x_{0}\right| \leq \epsilon_{0} \quad \text { if } \operatorname{dist}\left(t,\left\{\frac{n_{i}+n_{i+1}}{2}\right\}_{1 \leq i<p}\right) \leq 1 .
$$

So, as in the proof of Proposition 4.3, we can multiply $u$ by the cutoff functions $\Theta_{i}$ defined by formula (4.1). We recall that $\Theta_{i}=1$ on

$$
\left[\frac{n_{i}+n_{i-1}}{2}+1, \frac{n_{i+1}+n_{i}}{2}-1\right]
$$

and $\Theta_{i}=0$ outside

$$
\left[\frac{n_{i}+n_{i-1}}{2}-1, \frac{n_{i+1}+n_{i}}{2}+1\right]
$$

with the convention $n_{0}=-\infty, n_{p+1}=+\infty$. For $1 \leq i \leq p$, let

$$
\begin{gathered}
\operatorname{pr}_{i}: X_{n * K, \infty} \rightarrow \mathscr{E} \\
u \rightarrow \Theta_{i} u .
\end{gathered}
$$

By the uniform estimates of Proposition 4.3, the functions $\operatorname{pr}_{i}(u)$ converge to $X$ for the $H_{\text {semi-loc }}^{4,2}$-topology, as the distances $n_{i+1}-n_{i}$ between the bumps increase. Hence we find a constant $N_{0}$ such that $\operatorname{pr}_{i}(u) \in \mathcal{U}$ for all $u \in X_{n * K, \infty}$ with $n_{i+1}-n_{i} \geq 2 N_{0}$. 
Now let $\alpha_{1}, \ldots, \alpha_{p} \in \bar{H}^{*}(\Omega)$ be given as in the theorem, and let $n \in \mathbf{Z}^{p}$ satisfy $n_{i+1}-n_{i} \geq 2 N_{0}$ for all $i$. By the Künneth formula, the $\alpha_{i}$ give rise to a nonzero class

$$
\alpha_{1} \otimes \cdots \otimes \alpha_{p} \in \bigotimes_{i=1}^{p} \bar{H}^{*}(\Omega) \simeq \bar{H}^{*}\left(\Omega^{p}\right)
$$

In order not to get lost in notation, we identify

$$
\bigotimes_{i=1}^{p} \bar{H}^{*}(\Omega) \simeq \bar{H}^{*}\left(\Omega^{p}\right) \simeq \bar{H}^{*}\left(\Omega_{T}^{p}\right) \simeq \bar{H}^{*}\left(n * \Omega_{T}\right) \simeq \bar{H}^{*}\left(\tilde{\Omega}_{n}\right) .
$$

By assumption (a) on the $\alpha_{i}$ and Proposition 4.3,

$$
0 \neq\left(\left.\pi\right|_{X_{n * K, \infty}}\right)^{*}\left(\alpha_{1} \otimes \cdots \otimes \alpha_{p}\right) \in \bar{H}^{*}\left(X_{n * K, \infty}\right) .
$$

Now, let $\epsilon>0$ be given. Consider $u \in X_{n * K, \infty}$. If $n_{i+1}-n_{i} \geq 2 N(\epsilon)$ with $N(\epsilon)$ large enough, we can achieve, by Proposition 4.3 , that

$$
\left|u(s, t)-x_{0}\right| \leq \frac{\epsilon}{2} \quad \text { for } \operatorname{dist}\left(t,\left\{n_{i}\right\}_{1 \leq i<p}\right) \geq N(\epsilon)-1 .
$$

According to Corollary 3.6, there exists a connected component $\Gamma_{+}$of $\mathscr{C}$ such that $\operatorname{dist}_{L^{2}(\mathbb{R})}\left(u(s), \Gamma_{+}\right) \underset{s \rightarrow+\infty}{\longrightarrow} 0$ and $I\left(\Gamma_{+}\right)=I^{+}(u)$.

Let $u\left(s_{n}\right)$ be a subsequence converging to some $y \in \Gamma_{+}$. Using the cutoff functions $\Theta_{i}$ from above, we define $z_{i}:=\Theta_{i} y \in H^{1,2}(\mathbb{R}, T M)$. As $\min _{1 \leq i<p}\left(n_{i+1}-n_{i}\right) \rightarrow \infty$, each $z_{i}$ converges in $H^{1,2}(\mathbb{R}, T M)$ to a function $y_{i}$ with $I^{\prime}\left(y_{i}\right)=0$ (see [6, Proposition 2.8]). Hence if $N(\epsilon)$ is sufficiently large and $(\forall i) n_{i+1}-n_{i} \geq 2 N(\epsilon)$, then

$$
\left|z_{i}-y_{i}\right| \leq \frac{\epsilon}{2} \quad \text { for all } t \in \mathbb{R}
$$

Properties (i) and (iii) of the theorem follow from this. Now, since $I^{+}(u) \leq C$, we also have $I\left(y_{i}\right) \leq C$ for all $i$. So the theorem is proved if we can choose $u \in X_{n * K, \infty}$ such that $I^{+}\left(u_{i}\right) \geq m\left(\alpha_{i}\right)-\epsilon$ for all $i=1, \ldots, p$. Indeed, the $y_{1}, \ldots, y_{p}$ corresponding to such a $u$ satisfy $I\left(y_{i}\right) \geq m\left(\alpha_{i}\right)-\epsilon$. By Lemma 2.3 of [6], there exists a $\rho>0$ such that $I(x) \geq \rho$ for all homoclinic orbits (i.e., for all nontrivial critical points of $I$ ).

If $m\left(\alpha_{i}\right)$ was smaller than $\rho$, we would find a subset $A$ of $X$ with $\left(\left.\pi\right|_{A}\right)^{*} \alpha_{i} \neq 0$ and $\sup _{A} I^{+}<\rho$. But then all $u \in A$ would tend to $x_{0}$ as $s \rightarrow+\infty$ and therefore would be identically $x_{0}$; hence $A=\left\{x_{0}\right\}$ in contradiction with $\left(\left.\pi\right|_{A}\right)^{*} \alpha_{i} \neq 0, \alpha_{i} \in \bar{H}^{l_{i}}, l_{i}>0$. So $m\left(\alpha_{i}\right) \geq \rho$ for all $i$, which implies that $I\left(y_{i}\right)>0$ (for $\epsilon$ small enough); and we conclude that $y_{i}$ is a homoclinic orbit. Moreover, Proposition 4.3 gives two positive constants $c_{0}, \rho_{0}$ such that $y_{i}$ is in the compact set

$$
\mathscr{C}_{0}:=\left\{x \in \mathscr{b}:|x(t)| \leq c_{0} e^{-\rho_{0}|t|}\right\} .
$$


To prove the final statement, assume by contradiction that for every $u \in X_{n * K, \infty}$, there is a $j \in\{1, \ldots, p\}$ such that $I^{+}\left(u_{j}\right)<m\left(\alpha_{j}\right)-\epsilon$. More formally, define for pairs $B_{i} \subset A_{i}, 1 \leq i \leq p$, of topological spaces, the set

$$
\begin{aligned}
\Delta(A, B) & =\Delta\left(\left(A_{i}, B_{i}\right)_{1 \leq i \leq p}\right) \\
& =\bigcup_{i=1}^{p} A_{1} \times \cdots \times A_{i-1} \times B_{i} \times A_{i+1} \times \cdots \times A_{p} \\
& \subset \prod_{i=1}^{p} A_{i} .
\end{aligned}
$$

Then, with $U_{i}=\left\{u \in U: I^{+}(u) \leq m\left(\alpha_{i}\right)-\epsilon\right\}$ and

$$
\operatorname{pr}=\left(\operatorname{pr}_{1}, \ldots, \operatorname{pr}_{p}\right): X_{n * K, \infty} \rightarrow u^{p}
$$

the assumption reads as follows: $\operatorname{pr}\left(X_{n * K, \infty}\right) \subset \Delta\left(\left(\mathcal{U}, \mathcal{U}_{i}\right)_{1 \leq i \leq p}\right)$.

For paracompact Hausdorff spaces $A_{i}$ and closed subsets $B_{i} \subset A_{i}$, we have the following commutative diagram, where the horizontal lines are exact (see, e.g., [25]):

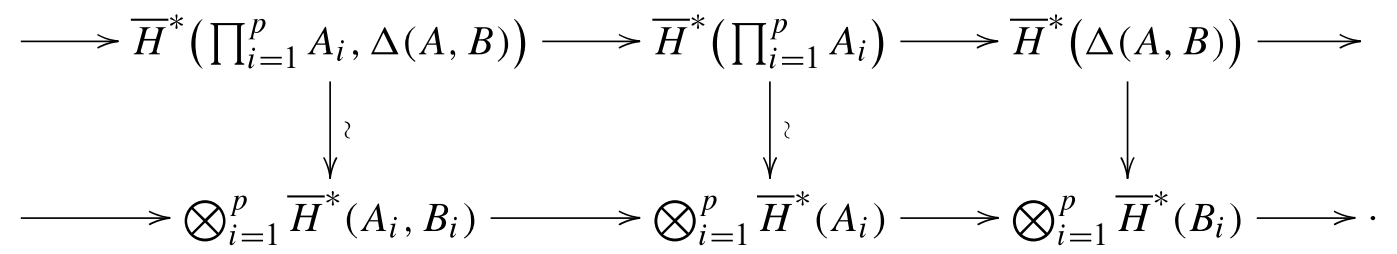

We see that if the image of an element of $\bigotimes_{i=1}^{p} \bar{H}^{*}\left(A_{i}\right)$ is zero in $\bigotimes_{i=1}^{p} \bar{H}^{*}\left(B_{i}\right)$, then also its image in $\bar{H}^{*}(\Delta(A, B))$ is zero.

We apply this to $A_{i}=U$ (which is a metric space, hence paracompact Hausdorff), $B_{i}=u_{i}$, and $\bigotimes_{i=1}^{p}(\pi \mid u)^{*} \alpha_{i} \in \bigotimes_{i=1}^{p} \bar{H}^{*}(u)$. By the definition of $m\left(\alpha_{i}\right)$ and the diagram above, we have

$$
0=\bigotimes_{i=1}^{p}\left(\pi \mid u_{i}\right)^{*} \alpha_{i} \in \bigotimes_{i=1}^{p} \bar{H}^{*}\left(u_{i}\right)
$$

Thus $0=\left(\left.\pi\right|_{\Delta}\right)^{*}\left(\alpha_{1} \otimes \cdots \otimes \alpha_{p}\right) \in \bar{H}^{*}\left(\Delta\left(\left(u, U_{i}\right)_{1 \leq i \leq p}\right)\right)$. But on the other hand, $\left.\pi\right|_{\Delta} \circ \mathrm{pr}$ is homotopic to $\left.\pi\right|_{X_{n * K, \infty}}$, and

$$
0 \neq\left(\left.\pi\right|_{X_{n K, \infty}}\right)^{*}\left(\alpha_{1} \otimes \cdots \otimes \alpha_{p}\right)=\operatorname{pr}^{*} \circ\left(\left.\pi\right|_{\Delta}\right)^{*}\left(\alpha_{1} \otimes \cdots \otimes \alpha_{p}\right) .
$$

This is a contradiction, and the theorem is proved.

Of course, Theorem 5.2 would be useless if there were no compact set $K \subset \Omega_{T}$ and classes $\alpha_{j} \in \bar{H}^{*}\left(\Omega, \mathbf{Z}_{2}\right)$ satisfying the hypotheses. Fortunately, by a theorem 
of Sullivan [26], the loop space of a simply connected compact manifold always has $\bar{H}^{l}\left(\Omega, \mathbf{Z}_{2}\right) \neq 0$ for some $l>0$. Now one just has to choose $K \subset \Omega_{T}$ large enough such that $i_{K}^{*}: \bar{H}^{l}(\Omega) \rightarrow \bar{H}^{l}(K)$ is injective (see [5]) and take nonzero classes $\alpha_{j} \in \bar{H}^{l}(\Omega)$. If $\pi_{1}(M) \neq 0$, one just takes nonzero $\alpha_{j} \in \bar{H}^{0}(\Omega)$ which vanish on the component of $\Omega$ consisting of the contractible loops. Then one can again conclude $m\left(\alpha_{j}\right) \geq \rho>0$, and Theorem 5.2 remains true in this case.

So Theorem 1.4 is proved under assumptions (H1), (H2), (H3), and (C), for $I$ a finite set. The case "I infinite" follows immediately from Ascoli's compactness lemma, since $N(\epsilon)$ does not depend on $p=\operatorname{Card}(I)$ (see [23]). The last statement of Theorem 1.4 about periodic orbits does not follow directly from Theorem 5.2 (see [9]). But it is easily obtained by studying the Cauchy-Riemann equation on cylinders $\mathbb{R} \times(\mathbb{R} / q \mathbf{Z}), q \in \mathbf{N}$ large, instead of $\mathbb{R} \times \mathbb{R}$, and by repeating the whole proof word by word.

To end the proof of Theorem 1.4 as a consequence of Theorem 5.2, we now explain how to remove the hypothesis $(\mathrm{H} 3)$.

Let $H$ satisfy (H1), (H2). Define a new Hamiltonian

$$
H_{A}(t, q, p)=f\left(\frac{|p|}{A}\right) \frac{|p|^{2}}{2}+\left(1-f\left(\frac{|p|}{A}\right)\right) H(t, q, p),
$$

where $f: \mathbb{R} \rightarrow[0,1]$ is smooth and such that

$$
f(s) \equiv 0 \quad \text { for } s \leq 1, \quad f(s) \equiv 1 \quad \text { for } s \geq 2,
$$

and $A \geq 1$ is a large constant. All $H_{A}$ 's, $A \geq 1$, satisfy (H1), (H2) with constants $a, b_{1}, b_{2}, c_{1}$, and $c_{2}$ independent of $A$. Moreover, all $H_{A}$ 's satisfy (H3) with a constant depending on $A$. Now choose a compact subset $K \subset \Omega_{T}$ and $p$ cohomology classes $\alpha_{1}, \ldots, \alpha_{p}$ with $i_{K}^{*}\left(\alpha_{j}\right) \neq 0$ as in Theorem 5.2. By Lemma 4.2(ii), there exists a constant $h>0$ such that

$$
I_{A}(x) \leq h
$$

for all $x \in H^{1,2}(\mathbb{R}, T M)$ with $\tau x \in \bar{n} * K, \bar{n}=\left(n_{1}, \ldots, n_{p}\right)$ and for all $A \geq 1$. Here $I_{A}$ denotes the action with Hamiltonian $H_{A}$.

On the other hand, if we denote by $\mathscr{C}_{A}$ the set of homoclinic orbits for $H_{A}$, we obtain from Lemma 4.1 that $\|x\|_{H^{1,2}}^{2} \leq K h$ for all $x \in \mathscr{C}_{A}$ with $I_{A}(x) \leq h$. Hence all such solutions satisfy $\|p\|_{L^{\infty}} \leq h^{\prime}$ with the same constant $h^{\prime}$ not depending on $A$. So if we take $A>h^{\prime}$, we get

$$
\left(x \in \mathscr{C}_{A}, I_{A}(x) \leq h\right) \Rightarrow x \in \mathscr{C} .
$$

In particular, if $H$ satisfies (C), then for $A>h^{\prime}$, all connected components of $\mathscr{C}_{A}^{h}=$ $\left\{x \in \mathscr{C}_{A} ; I_{A}(x) \leq h\right\}$ are compact. This allows us to carry out the proof of Theorem 5.2 for fixed $K, p,\left(\alpha_{1}, \ldots, \alpha_{p}\right)$ and a Hamiltonian $H_{A}, A>h^{\prime}$. We find $y_{1}, \ldots, y_{p}, y \in$ $\mathscr{C}_{A}^{h}$, with the properties described in Theorem 5.2. But as observed above, $y_{1}, \ldots, y_{p}, y$ 
are actually solutions for the original Hamiltonian $H$, and therefore Theorem 1.4 is proved in the general case.

\section{REFERENCES}

[1] V. Benci And F. Giannoni, Homoclinic orbits on compact manifolds, J. Math. Anal. Appl. 157 (1991), 568-576.

[2] U. Bessi, A variational proofofa Sitnikov-like theorem , Nonlinear Anal. 20 (1993), 1303-1318.

[3] S. V. Bolotin, Libration motions ofnatural dynamical systems (in Russian), Vestnik Moscov Univ. Ser. I Mat. Mekh. 1978, no. 6, 72-77, English translation in Moscow Univ. Mech. Bull. 33, no. 6 (1978), 49-53.

[4] B. BUFFONI AND E. SÉRÉ, A global condition for quasi-random behavior in a class of conservative systems, Comm. Pure Appl. Math. 49 (1996), 285-305.

[5] K. Cieliebak, Pseudo-holomorphic curves and periodic orbits on cotangent bundles, J. Math. Pures Appl. (9) 73 (1994), 251-278.

[6] K. CieliebAK AND E. SÉRÉ, Pseudoholomorphic curves and multiplicity ofhomoclinic orbits , Duke Math. J. 77 (1995), 483-518.

[7] V. Coti Zelati, I. Ekeland, And E. SÉRÉ, A variational approach to homoclinic orbits in Hamiltonian systems, Math. Ann. 288 (1990), 133-160.

[8] V. Coti Zelati And P. Rabinowitz, Homoclinic orbits for second order Hamiltonian systems possessing superquadratic potentials, J. Amer. Math. Soc. 4 (1991), 693-727.

[9] - Multibump periodic solutions ofa fimily ofHamiltonian systems , Topol. Methods Nonlinear Anal. 4 (1994), 31-57.

[10] P. Felmer, Heteroclinic orbits for spatially periodic Hamiltonian systems, Ann. Inst. H. Poincaré Anal. Non Linéaire 8 (1991), 477-497.

[11] A. Floer, The unregularized gradient flow ofthe symplectic action, Comm. Pure Appl. Math. 41 (1988), 775-813.

[12] F. GIANNONI AND P. RABINOWitz, On the multiplicity ofhomoclinic orbits on Riemannian manifolds for a class of second order Hamiltonian systems, NoDEA Nonlinear Differential Equations Appl. 1 (1994), 1-46.

[13] M. Gromov, Pseudoholomorphic curves in symplectic manifolds, Invent. Math. 82 (1985), 307-347.

[14] H. Hofer, Lusternik-Schnirelman-theory for Lagrangian intersections, Ann. Inst. H. Poincaré Anal. Non Linéaire 5 (1988), 465-499.

[15] H. HoFER AND K. WysOcKI, First order elliptic systems and the existence ofhomoclinic orbits in Hamiltonian systems, Math. Ann. 288 (1990), 483-503.

[16] P.-L. Lions, The concentration-compactness principle in the calculus ofvariations: The locally compact case, I, Ann. Inst. H. Poincaré Anal. Non Linéaire 1 (1984), 109-145; II, 223 283.

[17] P. Montechiarri, M. Nolasco, and S. Terracini, a global condition for multibump phenomena in Duffing-like equations, to appear in Trans. Amer. Math. Soc.

[18] J. Moser, Stable and Random Motions in Dynamical Systems, Ann. Math. Stud. 77, Princeton University Press, Princeton, 1973.

[19] H. Poincaré, Les méthodes nouvelles de la mécanique céleste, Vol. III Gauthier-Villars, Paris, 1899.

[20] P. Rabinowitz, Periodic and heteroclinic orbits for a periodic Hamiltonian system, Ann. Inst. H. Poincaré Anal. Non Linéaire 6 (1989), 331-346.

[21] - A multibump construction in a degenerate setting, Calc. Var. Partial Differential Equations 5 (1997), 159-182.

[22] E. SÉRÉ, Existence ofinfinitely many homoclinic orbits in Hamiltonian systems, Math. Z. 209 
(1992), 27-42.

[23] Looking for the Bernoulli shift, Ann. Inst. H. Poincaré Anal. Non Linéaire 10 (1993), 561-590.

[24] S. Smale, An infinite dimensional version ofSard's theorem, Amer. J. Math. 87 (1965), 861866.

[25] E. H. Spanier, Algebraic Topology, McGraw-Hill, New York, 1966.

[26] D. Sullivan, "Differential forms and the topology of manifolds" in Proc. Internat. Conf. on Manifolds and Related Topics in Topology (Tokyo, 1973), University of Tokyo Press, Tokyo, 1975, 37-49.

[27] W. P. Thurston, Travaux de Thurston sur les surfaces: Séminaire Orsay, Astérisque 66-67, Soc. Math. France, Paris, 1979.

Cieliebak: Mathematik, ETH-Zentrum, Rämistrasse 101, CH-8092 Zürich, Switzerland; kai.cieliebak@math.ethz.ch

Séré: Ceremade, Université Paris-Dauphine, Place du Maréchal de Lattre de Tassigny, 75775 PARIs CEDEX 16, FRANCE; sere@pi.ceremade.dauphine.fr 